\title{
Efficient Implementations of Rainbow and UOV using AVX2
}

\author{
Kyung-Ah Shim ${ }^{1 \dagger}$, Sangyub Lee ${ }^{1}$, Namhun Koo ${ }^{2}$ \\ ${ }^{1}$ National Institute for Mathematical Sciences, Daejeon, Republic of Korea, \\ \{kashim, sylee\}@nims.re.kr \\ ${ }^{2}$ Ewha Womans University, Seoul, Republic of Korea, \\ nhkoo@ewha.ac.kr
}

\begin{abstract}
A signature scheme based on multivariate quadratic equations, Rainbow, was selected as one of digital signature finalists for NIST Post-Quantum Cryptography Standardization Round 3. In this paper, we provide efficient implementations of Rainbow and UOV using the AVX2 instruction set. These efficient implementations include several optimizations for signing to accelerate solving linear systems and the Vinegar value substitution. We propose a new block matrix inversion (BMI) method using the Lower-Diagonal-Upper decomposition of blocks matrices based on the Schur complement that accelerates solving linear systems. Compared to UOV implemented with Gaussian elimination, our implementations with the BMI result in speedups of $12.36 \%, 24.3 \%$, and $34 \%$ for signing at security categories I, III, and $\mathrm{V}$, respectively. Compared to Rainbow implemented with Gaussian elimination, our implementations with the BMI result in speedups of $16.13 \%$ and $20.73 \%$ at the security categories III and V, respectively. We show that precomputation for the Vinegar value substitution and solving linear systems dramatically improve their signing. UOV with precomputation is 16.9 times, 35.5 times, and 62.8 times faster than UOV without precomputation at the three security categories, respectively. Rainbow with precomputation is 2.1 times, 2.2 times, and 2.8 times faster than Rainbow without precomputation at the three security categories, respectively. We then investigate resilience against leakage or reuse of the precomputed values in UOV and Rainbow to use the precomputation securely: leakage or reuse of the precomputed values leads to their full secret key recoveries in polynomial-time.
\end{abstract}

Keywords: Block Matrix Inversion - Digital Signature - Gaussian Elimination . Multivariate-Quadratic Problem · Post-Quantum Cryptography · Precomputation · Schur Complement

\section{Introduction}

Developments of a quantum computer have inspired great interest in post-quantum cryptographic primitives that are believed to remain secure against a quantum computer. Cryptographic primitives based on multivariate quadratic (MQ) equations are one of promising post-quantum replacements for current public-key cryptographic algorithms based on the discrete logarithm problem and the integer factorization problem. In 2020, NIST selected seven finalists for Post-Quantum Cryptography (PQC) Standardization Round $3\left[\mathrm{ABC}^{+} 20, \mathrm{SAB}^{+} 20, \mathrm{CDH}^{+} 20, \mathrm{DKR}^{+} 20, \mathrm{LDK}^{+} 20, \mathrm{PFH}^{+} 20, \mathrm{DCP}^{+} 20 \mathrm{~b}\right]$. The third-round finalists for digital signatures are two lattice-based schemes, Dilithium and

\footnotetext{
${ }^{\dagger}$ Corresponding Author. This research was supported by the National Institute for Mathematical Sciences funded by Ministry of Science and ICT of Korea (B21720000).
} 
Falcon, and an MQ-scheme, Rainbow. These finalists will be considered for standardization at the end of the third round.

MQ-schemes mainly rely on the hardness of solving large systems of multivariate quadratic equations, called the MQ-problem. To hide a trapdoor, they require an AffineSubstitution-Affine (ASA) structure related to the Extended Isomorphism of Polynomials (EIP) problem [Pat96]. Since Imai and Matsumoto [MI88] introduced the first MQencryption scheme, most of the MQ-schemes have been broken except few signature schemes including Unbalanced Oil-and-Vinegar (UOV) variants [KPG99, DS05] and HFEvvariants [PCG01, $\left.\mathrm{PCY}^{+} 15\right]$. MQ-schemes require only modest computational resources and are suitable for resource constrained devices [BERW08, $\mathrm{CCC}^{+} 09$ ]: it is easy to implement requiring simple operations such as matrix-vector products and solving linear systems over small finite fields without multi-precision arithmetic. Thus, they can be efficiently implemented on low cost devices, without the need of a cryptographic coprocessor. Their signature length and performance are the shortest and fastest among known post-quantum signature schemes, respectively. In spite of fast performance and short signatures, they suffer from relatively large key sizes. However, in medium devices with sufficient memory capabilities such as smartphones, large key size is not a major problem.

UOV and Rainbow are based on two hard problems, the MQ-problem and the EIPproblem, while Rainbow additionally requires the hardness of the MinRank problem due to its multi-layered structure. New methods to improve the MinRank attack are still being developed [PS20, $\left.\mathrm{VBC}^{+} 19, \mathrm{BBB}^{+} 20, \mathrm{BBC}^{+} 20\right]$. Rainbow team $\left[\mathrm{DCP}^{+} 20 \mathrm{a}\right]$ modified their parameters based on these new security analyses by increasing the number of equations and variables. Ward Beullens [Beu20] gave new intersection attacks on UOV and Rainbow, and MinRank attacks on Rainbow. He claimed that the attacks reduced the cost of a key recovery by a factor of $2^{16}, 2^{30}$, and $2^{46}$ for the parameter sets of Rainbow submitted to NIST PQC at three security categories, respectively. Subsequently, Rainbow team showed that the Rainbow parameters submitted to Round 3 still meet the NIST level I, III, and $\mathrm{V}$ security requirements in their updated security model and analysis without detracting from Ward's work $\left[\mathrm{DCP}^{+} 20 \mathrm{c}\right]$.

Main Results. A main idea to invert a system of quadratic equations in UOV and Rainbow is to convert the quadratic system to a linear system by substituting random Vinegar values into the Vinegar variables of the central quadratic equations. Signing of UOV and Rainbow is dominated by these two operations: the Vinegar value substitution and solving linear systems. In this paper, we present optimizations to accelerate the two major operations via a block matrix inversion method and precomputation.

- Implementations of UOV/Rainbow. We select new parameters of UOV secure against the recent attacks including known algebraic attacks at the three security categories. We then implement UOV with the new parameters and Rainbow with the modified parameters submitted to NIST PQC at the three security categories I, III, and $\mathrm{V}\left[\mathrm{DCP}^{+} 20 \mathrm{a}\right]$. After profiling their implementations, we determine target optimizations.

- Efficient Implementations of UOV/Rainbow. We propose two optimizations to improve signing of UOV and Rainbow, and present their efficient implementations in $\mathrm{C}$ program language on an Intel 64-bit processor using the AVX2 instruction set.

- Block Matrix Inversion (BMI) Method. We propose a BMI method using the Lower-Diagonal-Upper decomposition of block matrices based on the Schur complement. We use the BMI method to reduce the size of the matrix being inverted by half for accelerating solving linear systems. Compared to UOV implemented with Gaussian elimination, we speed up the signing by $12.36 \%$, $24.3 \%$, and $34 \%$ at the security categories I, III, and V, respectively. Compared 
to Rainbow implemented with Gaussian elimination, we speed up the signing by $16.13 \%$ and $20.73 \%$ at the security categories III and V, respectively.

- Precomputation. We present implementations of UOV and Rainbow with precomputation for message independent operations that dramatically improve signing. UOV with precomputation is 16.9 times, 35.5 times, and 62.8 times faster than UOV without precomputation at the three security categories, respectively, while Rainbow with precomputation is 2.1 times, 2.2 times, and 2.8 times faster than Rainbow without precomputation at the three security categories, respectively. Rainbow is faster than UOV, while UOV with precomputation is about 2.7 times, 7.3 times, and 12.2 times faster than Rainbow with precomputation at the three security categories, respectively.

- Resilience against Leakage or Reuse of the Precomputed Values. We investigate resilience of leakage or reuse of the precomputed values in UOV and Rainbow to use the precomputation values securely. We show that if $(n+1)$ signatures generated by the revealed precomputed values are given then the secret keys of UOV and Rainbow are completely recovered in polynomial-time. If $(m+1)$ and $\left(o_{2}+1\right)$ signatures generated by reusing the precomputed values are given then the secret keys of UOV and Rainbow are entirely recovered in polynomial-time, respectively.

Organization. Section 2 describes Rainbow and UOV specifications. In Section 3, we select new parameters of UOV secure against the recent attacks and we determine target optimizations after profiling their implementations. In Section 3, we present the BMI method for solving linear systems and investigate the actual improvements in terms of matrix sizes and the security levels. In Section 4, we propose UOV and Rainbow with precomputation and then investigate resilience against leakage or reuse of the precomputed values. In each section, we present efficient UOV/Rainbow implementations with our optimizations using the AVX2 instruction set and investigate their speedups compared to the original schemes. We conclude this paper in Section 5 .

\section{Preliminaries}

We describe key generation, signing and verification algorithms of Rainbow and UOV.

\subsection{Rainbow and UOV}

\section{Main Parameters.}

- $\mathbb{F}_{q}$ : the finite field of $q$ elements

- $m$ : the number of polynomials in the public key

- $v$ : the number of Vinegar variables

- $o$ : the number of Oil variables in UOV, $m=o$

- $o_{i}$ : the number of Oil variables in the $i$-th layer of Rainbow, $m=o_{1}+o_{2}$

- $n$ : the number of variables in the public key, $n=m+v$.

We describe Rainbow with two layers. Let $v, o_{1}$, and $o_{2}$ be positive integers such that $m=o_{1}+o_{2}$ and $n=v+m$. Define sets of integers

$$
V=\{1, \cdots, v\}, O_{1}=\left\{v+1, \cdots, v+o_{1}\right\}, O_{2}=\left\{v+o_{1}+1, \cdots, v+o_{1}+o_{2}\right\} .
$$


A central map $\mathcal{F}(\mathbf{x})=\left(\mathcal{F}^{(1)}(\mathbf{x}), \cdots, \mathcal{F}^{(m)}(\mathbf{x})\right)$ is a system of $m$ multivariate quadratic polynomials in $n$ variables $x_{1}, \cdots, x_{n}$ defined by

$$
\begin{gathered}
\mathcal{F}^{(i)}(\mathbf{x})=\sum_{s \in O_{1}, t \in V} \alpha_{s t}^{(i)} x_{s} x_{t}+\sum_{s, t \in V, s \leq t} \beta_{s t}^{(i)} x_{s} x_{t}+\sum_{s \in V \cup O_{1}} \gamma_{s}^{(i)} x_{t}+\eta^{(i)}, 1 \leq i \leq o_{1} \\
\mathcal{F}^{(i)}(\mathbf{x})=\sum_{s \in O_{2}, t \in V \cup O_{1}} \alpha_{s t}^{(i)} x_{s} x_{t}+\sum_{s, t \in V \cup O_{1}, s \leq t} \beta_{s t}^{(i)} x_{s} x_{t}+\sum_{s \in V \cup O_{1} \cup O_{2}} \gamma_{s}^{(i)} x_{t}+\eta^{(i)}, o_{1}+1 \leq i \leq m
\end{gathered}
$$

Two invertible affine maps $\mathcal{S}: \mathbb{F}_{q}^{m} \rightarrow \mathbb{F}_{q}^{m}$ and $\mathcal{T}: \mathbb{F}_{q}^{n} \rightarrow \mathbb{F}_{q}^{n}$ are needed to destroy the special structure of $\mathcal{F}$. A public key is given by $\mathcal{P}=\mathcal{S} \circ \mathcal{F} \circ \mathcal{T}$ and a secret key is $(\mathcal{S}, \mathcal{F}, \mathcal{T})$.

\section{Rainbow}

- $\operatorname{KeyGen}\left(1^{\lambda}\right)$. For a security parameter $\lambda$, output a public key as $P K=\mathcal{P}=\mathcal{S} \circ \mathcal{F} \circ \mathcal{T}$ and a secret key as $S K=(\mathcal{S}, \mathcal{F}, \mathcal{T})$.

- $\operatorname{Sign}(S K, \lambda, \mathrm{m})$. Given a message $\mathrm{m}$ and a collision-resistant hash function $\mathcal{H}$ : $\{0,1\}^{*} \rightarrow \mathbb{F}_{q}^{m}$,

- Choose a $\lambda$-bit random salt $r$, compute $\mathbf{h}=\mathcal{H}(\mathrm{m}, r) \in \mathbb{F}_{q}^{m}$ and $\mathbf{a}=\mathcal{S}^{-1}(\mathbf{h})$.

- Compute $\mathbf{b}=\mathcal{F}^{-1}(\mathbf{a})$, i.e. $\mathcal{F}(\mathbf{b})=\mathbf{a}$ as follows:

* In the first layer, select Vinegar values $\left(s_{1}, \cdots, s_{v}\right) \in \mathbb{F}_{q}^{v}$ at random and obtain a linear system of $o_{1}$ equations with $o_{1}$ unknowns $x_{v+1}, \cdots, x_{v+o_{1}}$ by substituting $\left(s_{1}, \cdots, s_{v}\right)$ into $o_{1}$ central polynomials $\mathcal{F}^{(k)}$ for $1 \leq k \leq o_{1}$. After that, find a solution $\left(s_{v+1}, \cdots, s_{v+o_{1}}\right)$ of the linear system using Gaussian elimination.

* In the second layer, obtain a linear system of $o_{2}$ equations with $o_{2}$ unknowns $x_{v+o_{1}+1}, \cdots, x_{n}$ by substituting $\left(s_{1}, \cdots, s_{v+o_{1}}\right)$ into $o_{2}$ central polynomials $\mathcal{F}^{(i)}$ for $o_{1}+1 \leq i \leq m$. After that, find a solution $\left(s_{v+o_{1}+1}, \cdots, s_{n}\right)$ of the linear system using Gaussian elimination. Then $\mathbf{b}=\left(s_{1}, \cdots, s_{n}\right)$.

* If one of the linear systems is not solvable, choose another random number $r^{\prime}$ and try again.

- Compute $\sigma=\mathcal{T}^{-1}(\mathbf{b})$ and output $\tau=(\sigma, r)$ as a signature on $\mathrm{m}$.

- $\operatorname{Verify}(P K, \mathrm{~m}, \tau)$. Given a signature $(\tau, \mathrm{m})$ and the public key $\mathcal{P}$, check the equality $\mathcal{P}(\sigma)=\mathcal{H}(\mathrm{m}, r)$. If the equality holds, output valid.

\section{uOV}

- $\operatorname{Key} \operatorname{Gen}\left(1^{\lambda}\right)$. For a security parameter $\lambda$, a public key is $P K=\mathcal{P}=\mathcal{F} \circ \mathcal{T}$ and a secret key is $S K=(\mathcal{F}, \mathcal{T})$. UOV does not require an affine map $S$ since all the central polynomials have the same form.

- $\operatorname{Sign}(S K, \mathrm{~m})$. It is the same as that of Rainbow's first layer.

- $\operatorname{Verify}(P K, \mathrm{~m}, \sigma)$. It is the same as that of Rainbow.

Algorithm 1, Algorithm 2, and Algorithm 3 specify key generation, signing, and verification of Rainbow submitted to NIST PQC Standardization Round 3 [DCP $\left.{ }^{+} 20 \mathrm{~b}\right]$. The description of UOV is consistent with a single layer version of Rainbow. Algorithm 4, Algorithm 5, and Algorithm 6 specify key generation, signing, and verification of UOV. Their signing and verification algorithms use the hash value $\mathcal{H}(\mathcal{H}(\mathrm{m}) \| r)$ instead of $\mathcal{H}(\mathrm{m}, r)$ for efficiency. 

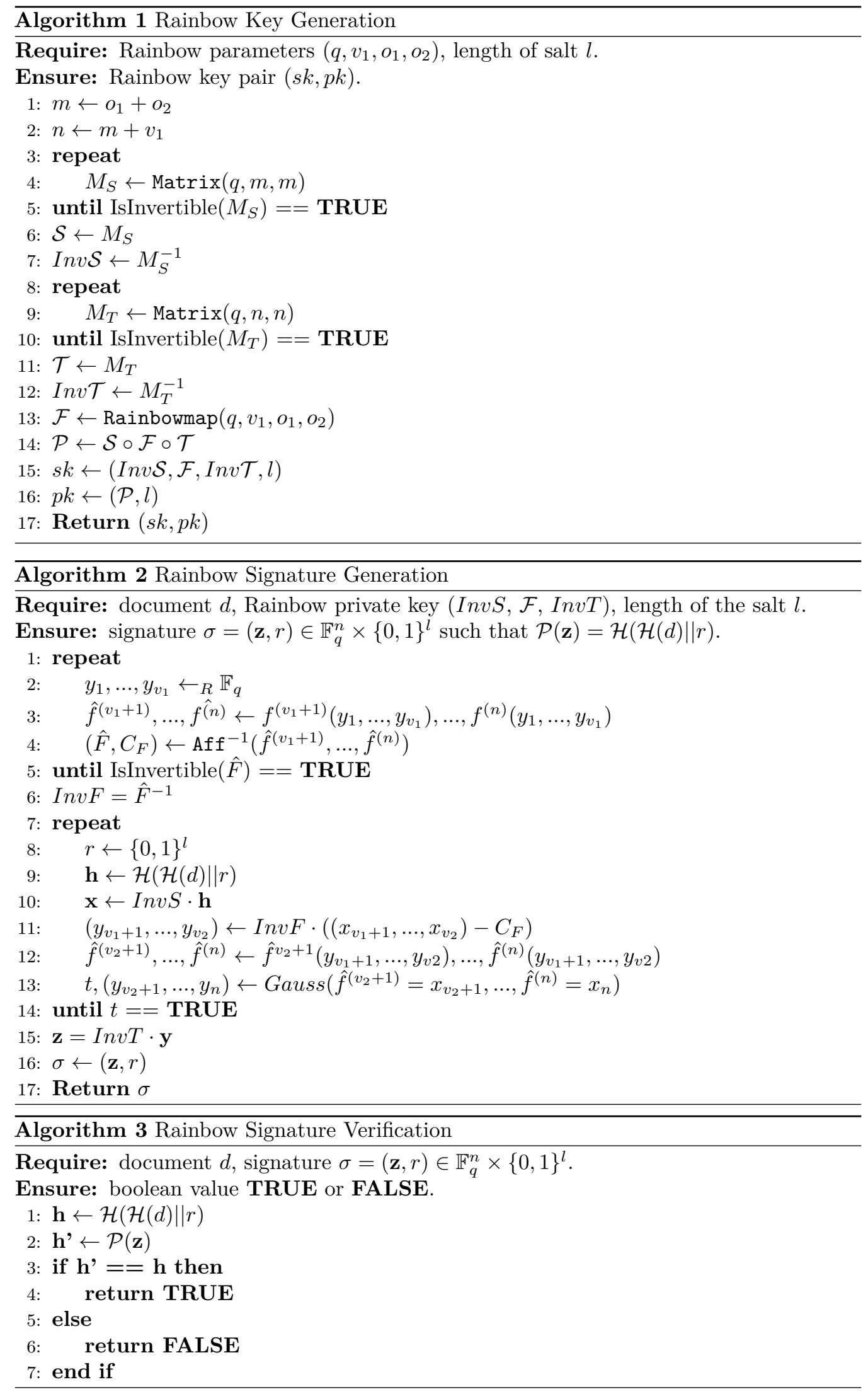

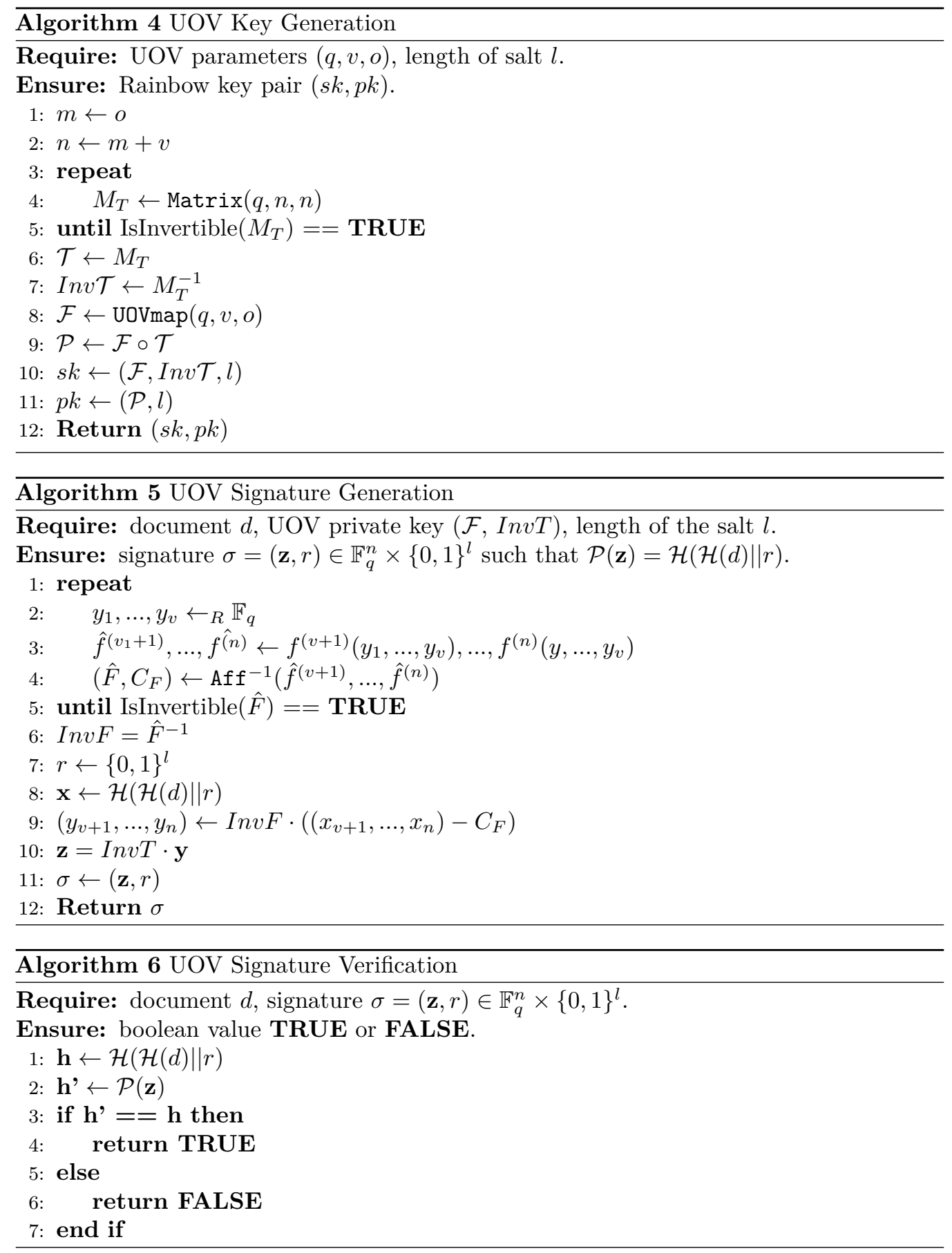

\section{Implementations of UOV and Rainbow}

We first consider major computations in UOV/Rainbow to improve their signing. We then select new secure parameters of UOV against the recent attacks including known algebraic attacks and implement them using the AVX2 instruction set. To identify how much improvement needs to be made in their implementations, we profile the implementations and determine optimization strategies. 


\subsection{Major Computations of UOV and Rainbow}

We consider major computations in UOV and Rainbow.

Major Computations in UOV and Rainbow. A main idea to invert a system of quadratic equations in UOV and Rainbow is to convert the quadratic system to a linear system by substituting random Vinegar values into the Vinegar variables of the central quadratic polynomials. There are two types of computations in signing:

- Substitution of Vinegar Values into the Central Polynomials. Calculations for substituting random Vinegar values into the central polynomials are required. Since there are a large number of quadratic terms with Vinegar $\times$ Vinegar indexes and Vinegar $\times$ Oil indexes being substituted by the Vinegar values, the computations are heavy.

- Solving Linear System. Solving the linear systems after the Vinegar value substitution are required. Gaussian elimination is used to find a solution of the linear system, whose complexity is $O\left(k^{3}\right)$ for a $k \times k$ random matrix.

Thus, signing requires $O\left(k^{3}\right)$ complexity, where $k$ is the number of equations in the linear system. These computations are the main bottlenecks for signing cost.

Parameters of UOV and Rainbow are given by $\left(\mathbb{F}_{q}, v, o\right)$, where $m=o$ and $n=v+m$, and $\left(\mathbb{F}_{q}, v, o_{1}, o_{2}\right)$, where $m=o_{1}+o_{2}$ and $n=v+m$, respectively. The parameters related to the computational burden are as follows:

- Large Numbers of Variables $(n)$. UOV and Rainbow require the large numbers of variables which cause heavy computational cost for the Vinegar value substitution.

- Large Sizes of Matrices being Inverted $\left(m, o_{2}\right)$. The numbers of equations $(m)$ in UOV and Rainbow are determined by the complexities against direct attacks. To solve the linear systems,

- UOV with a single layer requires one inversion of an $m \times m$ matrix, where $m(=o)$ is at most twice $o_{i}(i=1,2)$, where $o_{1}$ and $o_{2}$ are the numbers of equations in the first and second layers of Rainbow, respectively.

- Rainbow with two layers requires two inversions of an $o_{1} \times o_{1}$ matrix and an $o_{2} \times o_{2}$ matrix, where $m=o_{1}+o_{2}$, and $o_{i}$ is smaller than $o$ in UOV. In particular, according to the security analysis of Rainbow $\left[\mathrm{BBC}^{+} 20, \mathrm{DCP}^{+} 20 \mathrm{a}\right]$, a key formula for complexity includes the number $k$, which corresponds to $o_{2}$. The modified Rainbow parameters submitted to NIST PQC were chosen $o_{2}>o_{1}$ $\left[\mathrm{DCP}^{+} 20 \mathrm{a}\right]$. Since the increase of $k$ will increase the attack complexity, $o_{2}$ is getting bigger.

\subsection{Parameter Selection and Implementations of UOV and Rainbow}

Our implementations are based on the open source codes of Rainbow submitted to NIST PQC Standardization Round $3\left[\mathrm{DCP}^{+} 20 \mathrm{~b}\right]$. After implementing the schemes, we profile their implementations.

Target Platform. The computer we have used is equipped with an Intel(R) Core(TM) i9-10900X CPU running at the constant clock frequency of 3.70GHz.

Random Number Generation and Hashing. We use AES_CTR_DRBG as the random number generator. We use SHA-2 as the underlying hash function. In the SHA-2 
Table 1: Suggested parameters of UOV/Rainbow at three security categories.

\begin{tabular}{clrrr}
\hline Scheme & Security Category & \multicolumn{1}{c}{ I } & III & \multicolumn{1}{c}{ V } \\
\hline \multirow{4}{*}{ UOV } & \multicolumn{1}{c}{$\lambda($ Gates $)$} & $2^{146}$ & $2^{212}$ & $2^{274}$ \\
\cline { 2 - 5 } & $\left(\mathbb{F}_{q}, o, v\right)$ & $\left(\mathbb{F}_{2^{8}}, 46,70\right)$ & $\left(\mathbb{F}_{2^{8}}, 72,109\right)$ & $\left(\mathbb{F}_{2^{8}}, 96,144\right)$ \\
& Direct Attack & $2^{146.05}$ & $2^{212.05}$ & $2^{274.847}$ \\
& Intersection Attack & $2^{166.87}$ & $2^{236.36}$ & $2^{291.501}$ \\
\hline \multirow{5}{*}{ Rainbow } & $2^{147}$ & $2^{217}$ & $2^{281}$ \\
& \multicolumn{1}{c}{$\lambda($ Gates $)$} & $\left(\mathbb{F}_{2^{4}}, 36,32,32\right)$ & $\left(\mathbb{F}_{2^{8}}, 68,32,48\right)$ & $\left(\mathbb{F}_{2^{8}}, 96,36,64\right)$ \\
& Direct Attack & $\left.2^{164}, o_{2}\right)$ & $2^{234}$ & $2^{285}$ \\
& MinRank Attack & $2^{162}$ & $2^{228}$ & $2^{296}$ \\
& RBS Attack & $2^{147}$ & $2^{217}$ & $2^{281}$ \\
\hline
\end{tabular}

hash function family, we use SHA256, SHA384, and SHA512 with output lengths of 256, 384 , and 512 bits, respectively.

Selection of Finite Fields. We choose $\mathbb{F}_{q}=\mathbb{F}_{2^{8}}$ for UOV and $\mathbb{F}_{q}=\mathbb{F}_{2^{8}}$ or $\mathbb{F}_{2^{4}}$ for Rainbow as the underlying finite fields.

The Use of Random Salts. We use a random salts $r \in\{0,1\}^{l}$ to achieve provable security as in [SSH11] which should be used only once.

Use of Equivalent Key and Linear Maps. UOV implementation uses a secret key $\mathcal{T}$ as an equivalent key of UOV of the form $\mathcal{T}=\left(\begin{array}{cc}I & T^{\prime} \\ 0 & I\end{array}\right)$ and a linear map. Rainbow implementation uses a secret key $(\mathcal{S}, \mathcal{T})$ as an equivalent key of Rainbow of the form $\mathcal{S}^{-1}=\left(\begin{array}{cc}I & S^{\prime} \\ 0 & I\end{array}\right), \mathcal{T}=\left(\begin{array}{ccc}I & T^{(1)} & T^{(2)} \\ 0 & I & T^{(3)} \\ 0 & 0 & I\end{array}\right)$ and linear maps.

Selection of Secure Parameters. For implementations of UOV and Rainbow, we need to select their secure parameters.

- For UOV, we need to select new secure parameters of UOV against the attacks in [Beu20] including known algebraic attacks. In [CHT12], $\operatorname{UOV}\left(\mathbb{F}_{q}, o, v\right)=\left(\mathbb{F}_{2^{8}}, 44,59\right)$ was suggested as a secure parameter at a 128-bit security level. The intersection attack on $\operatorname{UOV}\left(\mathbb{F}_{2^{8}}, 44,59\right)$ [Beu20] requires only $2^{95}$ multiplications to recover the secret key, which is much less than the claimed security $2^{128}$. We suggest new secure parameters of UOV at the three security categories summarized in Table 1, where $\lambda$ is the required number of gates for each security category [ $\left.\mathrm{DCP}^{+} 20 \mathrm{~b}\right]$.

- For Rainbow, we use the modified parameters submitted to NIST at the three security categories $\left[\mathrm{DCP}^{+} 20 \mathrm{a}\right]$. The parameters at the three security categories are summarized in Table 1. Complexities in Table 1 are the lowest complexities that determine the corresponding security levels. RBS attack stands for the Rainbow Band Separation attack.

UOV/Rainbow Implementation Results. We provide implementations of UOV and Rainbow based on codes submitted to NIST PQC Standardization Round 3 [DCP ${ }^{+} 20 \mathrm{~b}$ ] using the AVX2 instruction set on the target platform. 
Table 2: UOV/Rainbow implementation results using AVX2 at three security categories in $\mathrm{CPU}$ cycles.

\begin{tabular}{rlrrr}
\hline Scheme & Security Category & \multicolumn{1}{c}{ I } & \multicolumn{1}{c}{ III } & \multicolumn{1}{c}{ V } \\
\hline \multirow{2}{*}{ UOV } & KeyGen. & 29077126 & 98870925 & 161016435 \\
& Sign & 201834 & 707959 & 1486775 \\
& Verify & 125312 & 222012 & 485344 \\
\hline \multirow{3}{*}{ Rainbow } & KeyGen. & 11575144 & 65099975 & 214977689 \\
& Sign & 68203 & 322799 & 807309 \\
& Verify & 46857 & 151466 & 395259 \\
\hline
\end{tabular}

- The results presented in Table 2 include the numbers of CPU cycles required by the key generation, signing, and verification.

- Each result is an average of 10,000 measurements for each function using the C programming language with GNU GCC version 10.1.0 compiler on Centos 7.9.2009. Hyperthreading and Turbo Boost are switched off.

Profiling UOV/Rainbow Implementation. To identify how much improvement needs to be made in the implementations, we profile their signing given in Table 3.

- In Table 3, Vinegar value substitution represents the process of substituting Vinegar values into the central polynomials, $\mathcal{F}^{(i)}$ for $i=1, \cdots, m$ in $\operatorname{UOV}\left(i=1, \cdots, o_{1}\right.$ and $i=o_{1}+1, \cdots, m$ in the first and second layers of Rainbow, respectively) after choosing random Vinegar values $s_{V}=\left(s_{1}, \cdots, s_{V}\right) \in \mathbb{F}_{q}^{v}$. An $m \times m$ matrix, $L S_{V}$, is the coefficient matrix of the linear system obtained from the Vinegar value substitution in UOV. Two $o_{1} \times o_{1}$ and $o_{2} \times o_{2}$ matrices, $L S_{V, 1}$ and $L S_{V, 2}$, are the coefficient matrices of the linear systems obtained from the Vinegar value substitution in the first and second layers of Rainbow, respectively. Etc. represents the rest of operations except the above two operations.

- Run-time of signing in UOV and Rainbow is mostly dominated by the two operations. In UOV, the proportions of cycles spent in the Vinegar values substitution and in finding an inverse matrix are up to $58 \%$ and $48 \%$, respectively. In Rainbow, the proportions of cycles spent in the two major operations are up to $63 \%$ and $42 \%$, respectively.

- Thus, optimizing these two operations are going to provide a large speedup.

- To accelerate solving linear systems, we will reduce the sizes of matrices being inverted by half based on a block matrix inversion method.

- We will use precomputation to handle both of the Vinegar value substitution and solving linear systems.

Timing Attack Protection. As in the implementation of Rainbow $\left[\mathrm{DCP}^{+} 19\right]$, all key dependent operations of our implementations of UOV/Rainbow are performed in a time-constant manner.

\section{Efficient Implementations of UOV and Rainbow}

In this section, we propose a block matrix inversion method and precomputation for solving linear systems and the Vinegar value substitution to accelerate signing of UOV 
Table 3: Profiling UOV/Rainbow implementations at three security categories.

\begin{tabular}{|c|c|c|c|c|c|}
\hline Scheme & Layer & Operations & $\mathrm{I}$ & III & $\mathrm{V}$ \\
\hline \multirow{3}{*}{ UOV } & \multirow{3}{*}{1} & Vinegar value substitution & $58.09 \%$ & $48.37 \%$ & $56.60 \%$ \\
\hline & & Computation of $L S_{V}^{-1}$ & $36.38 \%$ & $47.98 \%$ & $41.71 \%$ \\
\hline & & Etc. & $5.53 \%$ & $3.65 \%$ & $1.69 \%$ \\
\hline \multirow{6}{*}{ Rainbow } & \multirow{3}{*}{1} & Vinegar value substitution & $19.15 \%$ & $18.58 \%$ & $34.37 \%$ \\
\hline & & Computation of $L S_{V, 1}^{-1}$ & $27.04 \%$ & $11.37 \%$ & $8.03 \%$ \\
\hline & & Etc. & $3.49 \%$ & $1.26 \%$ & $0.68 \%$ \\
\hline & \multirow{3}{*}{2} & Vinegar value substitution & $24.08 \%$ & $39.54 \%$ & $29.07 \%$ \\
\hline & & Computation of $L S_{V, 2}^{-1}$ & $15.00 \%$ & $25.38 \%$ & $25.34 \%$ \\
\hline & & Etc. & $11.24 \%$ & $3.87 \%$ & $2.51 \%$ \\
\hline
\end{tabular}

and Rainbow. For our implementations, we utilize Rainbow team's AVX2-specialized basic functions for GF arithmetic, basic linear algebra, and matrix operations, and constanttime basic functions in its source code submitted to NIST PQC Round 3. For the BMI implementation, we use its AVX2 specialized functions for matrix operations and (constanttime) Gaussian elimination. Implementations for precomputation are constructed with rearranging the computational operations and adjusting the memory operations.

\subsection{A Fast Method for Solving Linear Systems: Block Matrix Inversion}

Signing of UOV and Rainbow uses Gaussian elimination to get $L S_{V}^{-1}$. The size of a matrix being inverted is one of the reasons for heavy computation which requires $O\left(\mathrm{~m}^{3}\right)$ complexity for inverting an $m \times m$ matrix. Now, we propose a new method, block matrix inversion method, to reduce the size of a matrix being inverted for accelerating UOV/Rainbow signing. We then investigate the actual improvements of our method in their implementations at various security levels.

Reduce the Size of a Matrix being Inverted. We first consider a nonsingular square matrix $R$ and its inverse $R^{-1}$ that can be partitioned into $2 \times 2$ blocks as

$$
R=\left(\begin{array}{cc}
A & B \\
C & D
\end{array}\right) \text { and } R^{-1}=\left(\begin{array}{cc}
E & F \\
G & H
\end{array}\right)
$$

The matrices $R$ and $R^{-1}$ must have even dimension in the all-square partitions. We consider the square diagonal partition of $R$ and $R^{-1}$. In this case, $A, D, E, H$ are square matrices, $A$ and $E$ have the same size, and so do $D$ and $H$. We use the following well-known theorem $\left[\mathrm{ND}^{+} 77\right]$.

Theorem 1. Let $R$ be a matrix partitioned into $2 \times 2$ blocks.

(i) Assume $A$ is nonsingular: then the matrix $R$ is invertible if and only if the Schur complement $\left(D-C A^{-1} B\right)$ of $A$ is invertible and

$$
R^{-1}=\left(\begin{array}{cc}
A^{-1}+A^{-1} B\left(D-C A^{-1} B\right)^{-1} C A^{-1} & -A^{-1} B\left(D-C A^{-1} B\right)^{-1} \\
-\left(D-C A^{-1} B\right)^{-1} C A^{-1} & \left(D-C A^{-1} B\right)^{-1}
\end{array}\right) .
$$

(ii) Assume $D$ is nonsingular: then the matrix $R$ is invertible if and only if the Schur complement $\left(A-B D^{-1} C\right)$ is invertible and 


$$
R^{-1}=\left(\begin{array}{cc}
\left(A-B D^{-1} C\right)^{-1} & -\left(A-B D^{-1} C\right)^{-1} B D^{-1} \\
-D^{-1} C\left(A-B D^{-1} C\right)^{-1} & D^{-1}+D^{-1} C\left(A-B D^{-1} C\right)^{-1} B D^{-1}
\end{array}\right)
$$

The inversion method in Theorem 1 requires two inversions for $A^{-1}$ and $\left[D-C A^{-1} B\right]^{-1}$, six matrix multiplications of the half-sized block matrices and three additive operations. To reduce the required operations, we use the Lower-Diagonal-Upper (LDU) decomposition of block matrices based on the Schur complement. We give an efficient block matrix inversion method for computing $R^{-1} \cdot \alpha$ to reduce the operations by more than half in Theorem 2 .

Theorem 2. For a nonsingular $k \times k$ matrix $R$ in the above, $R^{-1} \cdot \alpha$ requires two inversions, two matrix multiplications of the half-sized block matrices and four block matrix-vector products, where $k$ is even and $\alpha=\left(\alpha_{1}, \cdots, \alpha_{k / 2}\right)^{\mathrm{T}}$.

Proof. A nonsingular square matrix $R$ of $2 \times 2$ blocks is represented by the LDU decomposition of block matrices based on the Schur complement as

$R=\left(\begin{array}{cc}A & B \\ C & D\end{array}\right)=\left(\begin{array}{cc}I & O \\ C A^{-1} & I\end{array}\right)\left(\begin{array}{cc}A & O \\ 0 & D-C A^{-1} B\end{array}\right)\left(\begin{array}{cc}I & A^{-1} B \\ 0 & I\end{array}\right)=L \cdot D_{S c} \cdot U$.

Thus, $R^{-1}$ can be expressed by $A^{-1}$ and the inverse of the Schur complement of $A$, $\left[D-C A^{-1} B\right]^{-1}$, if they exist,

$$
\begin{gathered}
R^{-1}=U^{-1} \cdot D_{S c}^{-1} \cdot L^{-1} \\
=\left(\begin{array}{cc}
I & -A^{-1} B \\
0 & I
\end{array}\right)\left(\begin{array}{cc}
A^{-1} & O \\
0 & {\left[D-C A^{-1} B\right]^{-1}}
\end{array}\right)\left(\begin{array}{cc}
I & 0 \\
-C A^{-1} & I
\end{array}\right) .
\end{gathered}
$$

For computing $R^{-1} \cdot \alpha=R^{-1} \cdot\left(\begin{array}{c}\alpha_{1} \\ \cdots \\ \alpha_{k / 2}\end{array}\right)$, we reduce the matrix multiplications of the method in Theorem 1 as follows: After computing $A^{-1} B$ and $C\left(A^{-1} B\right)$, calculate $A^{-1}$ and $\left[D-C A^{-1} B\right]^{-1}$, where two matrix multiplications and two inversions of the block matrices. All remaining computations are made by four block matrix-vector products. We get

$$
R^{-1} \cdot\left(\begin{array}{c}
\alpha_{1} \\
\cdots \\
\alpha_{k / 2}
\end{array}\right)=\left(\begin{array}{cc}
I & -A^{-1} B \\
0 & I
\end{array}\right)\left(\begin{array}{cc}
A^{-1} & O \\
0 & {\left[D-C A^{-1} B\right]^{-1}}
\end{array}\right)\left(\begin{array}{cc}
I & 0 \\
-C A^{-1} & I
\end{array}\right)\left(\begin{array}{c}
\alpha_{1} \\
\cdots \\
\alpha_{k / 2}
\end{array}\right)
$$

We obtain $\left(C A^{-1}\right) \cdot\left(\begin{array}{c}\alpha_{1} \\ \cdots \\ \alpha_{k / 2}\end{array}\right)$ by computing $C\left[A^{-1} \cdot\left(\begin{array}{c}\alpha_{1} \\ \cdots \\ \alpha_{k / 2}\end{array}\right)\right]$ as two block matrix-vector products. Let $C A^{-1} \cdot\left(\begin{array}{c}\alpha_{1} \\ \cdots \\ \alpha_{k / 2}\end{array}\right)=\left(\begin{array}{c}\beta_{1} \\ \cdot \\ \beta_{k / 2}\end{array}\right)$. Next, we compute

$$
\left[D-C A^{-1} B\right]^{-1} \cdot\left(\begin{array}{c}
\beta_{1} \\
\cdots \\
\beta_{k / 2}
\end{array}\right)=\left(\begin{array}{c}
\gamma_{1} \\
\cdots \\
\gamma_{k / 2}
\end{array}\right),\left(A^{-1} B\right) \cdot\left(\begin{array}{c}
\gamma_{1} \\
\cdots \\
\gamma_{k / 2}
\end{array}\right)
$$

since $\left[D-C A^{-1} B\right]^{-1}$ and $A^{-1} B$ are already calculated. Thus, computing $R^{-1} \cdot \alpha$ requires two block matrix multiplications for computing $A^{-1} B$ and $C\left(A^{-1} B\right)$, two inversions for 
Table 4: Gaussian elimination (GE) vs. Block matrix inversion (BMI) method in CPU cycles.

\begin{tabular}{crcc}
\hline Matrix Size & \multicolumn{1}{c}{ GE } & BMI (Depth 1) & BMI (Depth 2) \\
\hline 46 & 94033 & 72302 & - \\
48 & 101498 & 75136 & 72479 \\
50 & 142243 & 82768 & - \\
56 & 175195 & 103357 & 99445 \\
64 & 225081 & 87150 & 70091 \\
68 & 322315 & 187947 & 170788 \\
72 & 355173 & 208355 & 190480 \\
96 & 713462 & 252538 & 226627 \\
100 & 923489 & 453441 & 391747 \\
\hline
\end{tabular}

$A^{-1}$ and $\left[D-C A^{-1} B\right]^{-1}$, and four block matrix-vector products. Its complexity is reduced to $O\left((k / 2)^{3}\right)$. Consequently, we reduce from six block matrix multiplications to two block matrix multiplications and four block matrix-vector products.

It is known that the naive way to perform a matrix multiplication requires $O\left(k^{3}\right)$ operations and Strassen's algorithm for matrix multiplications requires $O\left(k^{\log _{2} 7}\right)=O\left(k^{2.81}\right)$ operations [Str69]. We do not use Strassen's algorithm for matrix multiplications to compute $R^{-1} \cdot \alpha$ since Strassen's algorithm based on $2 \times 2$ block matrices makes the implementations very slow for larger matrices due to its recursive nature, and our method requires only two block matrix multiplications.

Repeated BMI: Determine the Minimum Size of a Matrix being Inverted. We want to determine the minimum size of a matrix being inverted.

- After performing the BMI, we reduced the size of a matrix being inverted by half, while the number of inversions for the half-sized matrices increased to two. We can apply the BMI again to these two half-sized matrices which results in four inversions of $k / 4 \times k / 4$ matrices and extra operations.

- Like this, for $m=2^{l} \cdot m^{\prime}$, we can apply the BMI $l$ times. We define the number of these iterations of the BMI as a depth. We cannot expect that $l$ iterations will always be effective, because $2^{l}$ inversions of $k / 2^{l} \times k / 2^{l}$ matrices are required.

Compatibility of the BMI Method with UOV and Rainbow. Unlike defined in the Rainbow specification [DCP ${ }^{+} 20 \mathrm{~b}$, our BMI method computes $L S_{V}^{-1} \cdot$ a directly without finding $L S_{V}^{-1}$. When using the BMI method for Rainbow (resp. UOV), if the block matrices of $L S_{V}^{-1}$ (resp., $L S_{V, 1}^{-1}$ or $L S_{V, 2}^{-1}$ ) are not invertible then new Vinegar values need to be chosen. Thus, to apply our BMI method to the signing algorithms of UOV and Rainbow, the specification of Rainbow and UOV would need to be changed from Algorithm 2 and 5 to Algorithm 7 and 8, respectively. In Algorithm 7 and 8, BMI(.) takes $L S_{V}$ and a vector as inputs then outputs a solution and a flag indicating $L S_{V}$ is solvable similar to Gauss(.) in Algorithm 2. Since the loop structure for each UOV/Rainbow signature generation algorithm is changed and random number generations for Vinegar and salt are involved within the loop, resulting signatures deploying the BMI method can be different from the specification although they are valid signatures) even if the seeds for random number generators as well as documents and secret keys are identical at the initial point of the algorithms. 

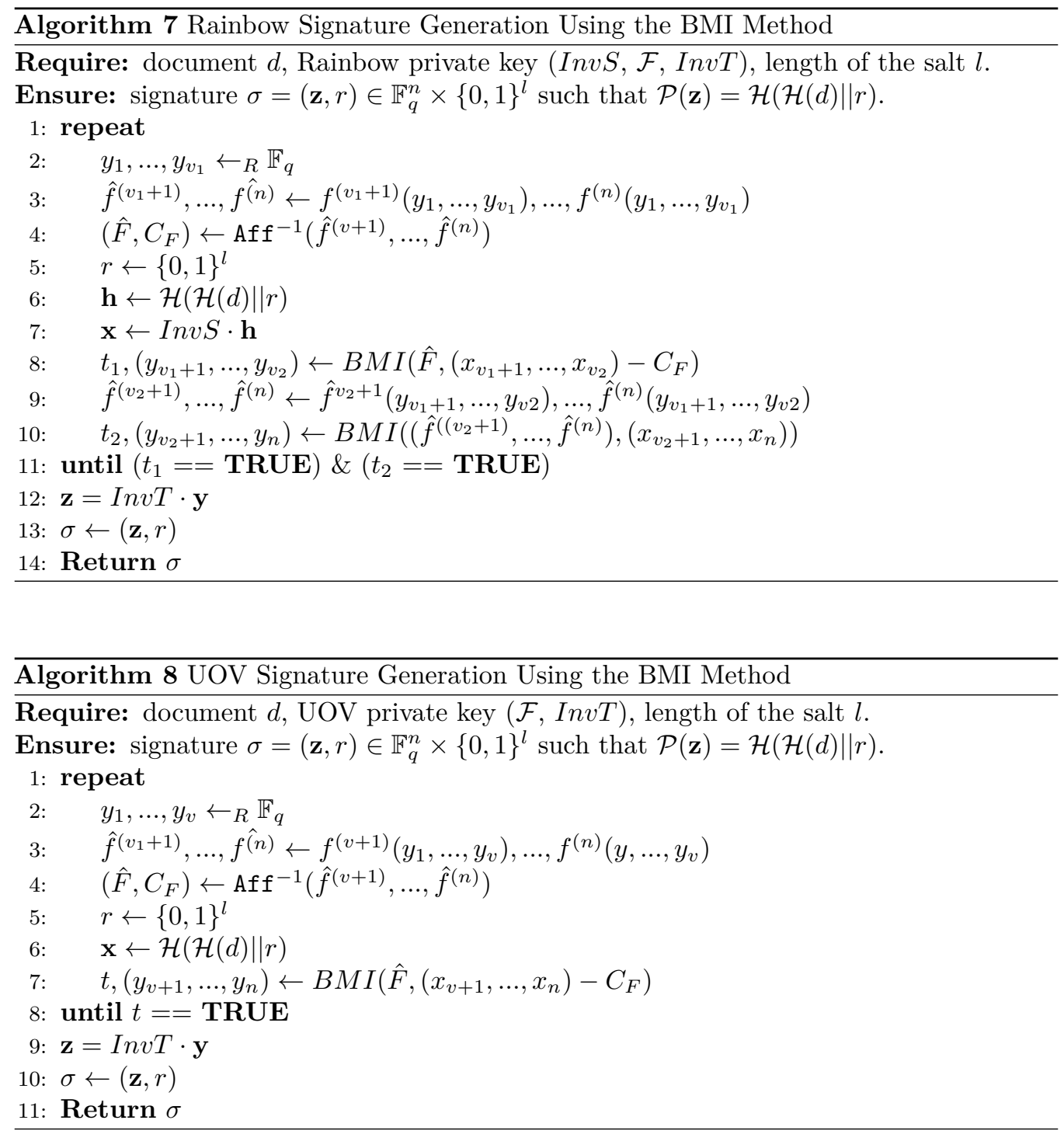

Implementation Results. We investigate improvements of UOV/Rainbow signing with the BMI method. For our implementations, we utilized Rainbow team's AVX2-specialized basic functions for GF arithmetic, basic linear algebra, and matrix operations in its source code submitted to NIST PQC Round 3. We used Rainbow team's AVX2 specialized functions for matrix operations and Gaussian elimination for the BMI implementation. The implementations of precomputation are constructed with rearranging computational operations and adjusting memory operations. We summarize their implementation results in terms of the size of matrices being inverted in Table 4 and in terms of the security categories in Table 5 .

- To use the BMI with the depth 1 and the depth 2, matrix sizes should be multiples of 2 and multiples of 4 , respectively. In the case of $m=46$, we cannot use the BMI with the depth 2 since it is not a multiple of 4 . We implement the BMI on the finite field $\mathbb{F}_{2^{8}}$. After obtaining $L S_{V}$ from the Vinegar value substitution, we set $L S_{V}=\left(\begin{array}{cc}A & B \\ C & D\end{array}\right)$, if $A$ or $\left[D-C A^{-1} B\right]$ is not invertible then we choose another Vinegar values. The probability that the matrices are invertible is $99.223 \%$. 
Table 5: UOV/Rainbow implementation results with the BMI method at three security categories in CPU cycles.

\begin{tabular}{clccr}
\hline Scheme & Security Category & I & III & V \\
\hline \multirow{3}{*}{ UOV } & Gaussian elimination & 201834 & 707959 & 1486775 \\
& BMI (Depth 1) & 176884 & 563519 & 1004704 \\
& BMI (Depth 2) & - & 535660 & 981351 \\
\hline \multirow{3}{*}{ Rainbow } & Gaussian elimination & 68203 & 322799 & 807309 \\
& BMI (Depth 1) & - & 270731 & 650400 \\
& BMI (Depth 2) & - & 271986 & 639965 \\
\hline
\end{tabular}

- As seen in Table 4 and Table 5, compared to Gaussian elimination, the larger the size, the greater the performance improvement and the higher the security category, the greater the effect.

- By using BMI with the depth 1, we get speedups of $23.1 \%, 41 \%, 64.6 \%$, and $50.9 \%$ at the sizes, $46,72,96$, and 100, respectively. Especially excellent improvements of $61.3 \%$ and $64.6 \%$ in the case of 64 and 96 , respectively, are due to the fact that the multiples of 32 are optimal parameters which are suitable for the AVX2 vectorization.

- In the BMI with the depth 2, their speedups are $46.54 \%, 68.23 \%$, and $57.58 \%$ at the sizes, 72,96 , and 100 , respectively.

- Compared to UOV implemented with Gaussian elimination, by using the BMI with the depth 1, we obtain speedups of $12.36 \%, 20.41 \%$, and $32.42 \%$ at the three security categories, respectively. For the BMI with the depth 2, the corresponding speedups are $24.3 \%$ and $34 \%$ at the security categories III and V, respectively. Compared to the BMI with the depth 1 , the effect of the BMI with the depth 2 is insignificant.

- Compared to Rainbow implemented with Gaussian elimination, by using BMI with the depth 1 , we obtain speedups $16.13 \%$ and $19.44 \%$ at the security categories III and V, respectively. For the BMI with depth 2, the corresponding speedup is $15.74 \%$ and $20.72 \%$ at the security categories III and V, respectively. Our results show that the BMI with larger depths are not always more efficient. Our BMI method in Rainbow implementation at the security category I is not effective. Since its parameter $\left(\mathbb{F}_{2^{4}}, 36,32,32\right)$ is very optimal, the implementation with Gaussian elimination is faster than that with the BMI method.

\subsection{Splitting Signing into Offline and Online Phase}

To speed up the signing process even more, we can choose to split the signature generation in an offline and online phase, where the offline phase can already be performed before the message to be signed is known. In 1989, Even, Goldreich, and Micali [EGM90] introduced the general idea of using an offline/online phase. Most costly message independent computations are completed in the offline phase and the device is idle. Such precomputation enables the online phase to quickly sign the messages with only light computation. Now, we describe the precomputation parts of UOV/Rainbow signing and evaluate the performance of offline/online computations using the AVX2 instruction set. 


\subsubsection{UOV with Precomputation}

Unlike Rainbow, UOV has significantly large message independent operations in signing. This offline precomputation can dramatically improve signing in UOV.

Signing. Signing can be divided into two parts: one is independent of messages being signed, the other depends on the messages.

- After substituting $s_{V}=\left(s_{1}, \cdots, s_{v}\right)$ into o equations $\mathcal{F}^{(k)}(1 \leq k \leq o)$, one gets a linear system of $o$ equations and $o$ variables $x_{v+1}, \cdots, x_{v+o}$ and computes $L S_{V}^{-1}$. Thus, $<s_{V}=\left(s_{1}, \cdots, s_{V}\right), c_{V}=\left(c_{1}, \cdots, c_{m}\right), L S_{V}^{-1}>$ can be precomputed, where $c_{V}$ is a vector of constant terms of $\left(\mathcal{F}^{(1)}\left(s_{V}\right), \cdots, \mathcal{F}^{(m)}\left(s_{V}\right)\right)$.

- This precomputation speedups the signing performance. However, it requires an additional computational overhead and one has to decide when to calculate and where to keep precomputed values. We will discuss how to use the precomputed values securely in the next subsection.

\section{Offline Signing of UOV.}

- After choosing random Vinegar values $s_{V}=\left(s_{1}, \cdots, s_{v}\right) \in \mathbb{F}_{q}^{v}$, substitute $s_{V}$ into $o$ equations $\mathcal{F}^{(k)}(1 \leq k \leq o)$ to get the linear system $L S_{V}$ of $o$ equations and $o$ unknowns and a constant vector $c_{V}=\left(c_{1}, \cdots, c_{m}\right)$.

- Compute $L S_{V}^{-1}$. If $L S_{V}$ is not invertible then go back to the first step.

- Store $<s_{V}, c_{V}, L S_{V}^{-1}>$ as the precomputed values.

\section{Online Signing of UOV.}

- Choose a random salt $r$ and compute $h=\mathcal{H}(\mathcal{H}(\mathrm{m}) \| r)$ for a message $\mathrm{m}$.

- From $<s_{V}=\left(s_{1}, \cdots, s_{V}\right), c_{V}=\left(c_{1}, \cdots, c_{m}\right), L S_{V}^{-1}>$, compute $L S_{V}^{-1} \cdot h_{V}^{\mathrm{T}}=\alpha$, where $h_{V}=\left(h_{1}-c_{1}, \cdots, h_{m}-c_{m}\right)$ and $h=\left(h_{1}, \cdots, h_{m}\right)$.

- Compute $T^{-1} \cdot\left(S_{V}, \alpha\right)^{\mathrm{T}}=\sigma$ and output $\tau=(\sigma, r)$ as a signature on $\mathrm{m}$.

\subsubsection{Rainbow with Precomputation}

In the first layer of Rainbow, the same precomputation is possible, but in the second layer, precomputation is limited since the vector consisting of the random Vinegar values and the solution of the linear system related to the message is supposed to be substituted into the central polynomials of the second layer. Hence, with the precomputed values for the first layer and some possible part of the second layer, computing a solution of the linear system from the first layer and then substituting the solution to the central polynomials and solving the linear system in the second layer are required for the online phase.

\section{Offline Signing of Rainbow.}

- After choosing random Vinegar values $s_{V}=\left(s_{1}, \cdots, s_{v}\right) \in \mathbb{F}_{q}^{v}$, substitute $s_{V}$ into $o_{1}$ equations $\mathcal{F}^{(k)}\left(1 \leq k \leq o_{1}\right)$ to get the linear system $L S_{V, 1}$ of $o_{1}$ equations and $o_{1}$ unknowns and a vector $c_{V, 1}=\left(c_{1}, \cdots, c_{o_{1}}\right)$ of constant terms $\left(\mathcal{F}^{(1)}\left(s_{V}\right), \cdots, \mathcal{F}^{\left(o_{1}\right)}\left(s_{V}\right)\right)$.

- Compute $L S_{V, 1}^{-1}$. If $L S_{V, 1}$ is not invertible then go back to the first step.

- Substitute $s_{V}$ into the Vinegar variables in $o_{2}$ equations $\mathcal{F}^{(k)}\left(o_{1}+1 \leq k \leq o_{1}+o_{2}\right)$ by getting a linear system of $o_{2}$ equations and unknowns, $\left\{\mathcal{F}^{\left(o_{1}+i\right)}\left(s_{V}\right)\right\}_{i=1}^{o_{2}}$. 
- Store $<s_{V, 1}, c_{V, 1}, L S_{V, 1}^{-1},\left\{\mathcal{F}^{\left(o_{1}+i\right)}\left(s_{V}\right)\right\}_{i=1}^{o_{2}}>$.

\section{Online Signing of Rainbow.}

- Compute $S^{-1} \cdot h^{\mathrm{T}}=\alpha$ for $h=\mathcal{H}(\mathcal{H}(m) \| r)$ with a message m and a random salt $r$.

- From $<s_{V, 1}, c_{V, 1}, L S_{V, 1}^{-1},\left\{\mathcal{F}^{\left(o_{1}+i\right)}\left(s_{V}\right)\right\}_{i=1}^{o_{2}}>$, compute $L S_{V, 1}^{-1} \cdot \alpha^{\mathrm{T}}=\delta$, where $\alpha=$ $\left(\alpha_{1}-c_{1}, \cdots, \alpha_{o_{1}}-c_{o_{1}}\right)$ and $\alpha=\left(\alpha_{1}, \cdots, \alpha_{o_{1}+o_{2}}\right)$.

- Compute $L S_{V, 2}^{-1}$, where $L S_{V, 2}$ is the coefficient matrix of the linear system obtained by substituting $\alpha$ into $o_{1}$-oil variables in $\left\{\mathcal{F}^{\left(o_{1}+i\right)}\left(s_{V}, \delta\right)\right\}_{i=1}^{o_{2}}$. If $L S_{V, 2}$ is not invertible then select another salt and go back to the first step.

- Compute $L S_{V, 2}^{-1} \cdot\left(\alpha_{o_{1}+1}, \cdots, \alpha_{o_{1}+o_{2}}\right)^{\mathrm{T}}=\zeta$ and $T^{-1} \cdot\left(S_{V}, \delta, \zeta\right)^{\mathrm{T}}=\sigma$, and output $\tau=(\sigma, r)$ as a signature on $\mathrm{m}$.

Implementation Results. Table 6 lists the benchmarking results of our UOV/Rainbow implementations with and without precomputation in cycle counts and required additional memory cost per signature.

- In Table 6, Sign w/ Precomp. and Sign w/o Precomp. represent signing with precomputation and signing without precomputation, respectively. Precomp. Memory Cost per Sig. represents the memory cost required for precomputation per signature.

- Compared to UOV without precomputation, UOV with precomputation obtains speedups of $94 \%, 97 \%$, and $98.43 \%$ at the three security categories, respectively.

- In Rainbow, the large portion of the second layer operations have to be done in the online phase. Compared to Rainbow without precomputation, Rainbow with precomputation obtains speedups of $52.89 \%, 55.16 \%$, and $64.32 \%$ at the three security categories, respectively.

- UOV with precomputation is about 2.7 times, 7.3 times and 12.2 times faster than Rainbow with precomputation at the three security categories, respectively.

- The results of Table 6 are without using the BMI method. The BMI method and precomputation can not be used together. The goal of the BMI method is to compute $L S_{V}^{-1} \cdot \alpha$, not $L S_{V}^{-1}$. A message is needed to compute $L S_{V}^{-1} \cdot \alpha$, where $L S_{V}$ is determined by the Vinegar values and a vector $\alpha$ is determined by a message being signed. Thus, to use the BMI method, the message is required, in that case, precomputation for message-independent operations is not possible.

Signing of Rainbow is very fast, but the higher the security level, the slower the performance. Thus, precomputation is suitable for all applications where maintaining fast performance is required even at the high security levels. Also, precomputation-based schemes fit into applications deploying the nodes with cost-effective energy harvesting technologies. The cost-effective energy harvesting technologies in WSNs are devised to supplement the battery power with energy gathered from the environment (e.g. solar, wind) and energy peaks are occasionally available. In some cases, the available energy can be even excessive, i.e. greater than the amount that can fit into the sensor node's supercapacitor, and thus it would be wasted unless used immediately [WWCJ10]. In this case, precomputation can be done for efficient use of energy.

Compared to recent precomputation results of Dilithium (lattice-based post-quantum signature scheme) in [RGCB19], Dilithium 3 with precomputation speedups upto $35 \%$ and requires an additional $260 \mathrm{KiB}$ of space for the precomputed values per signature. Furthermore, in Dilithium's precomputation, there was a $95 \%$ probability that at least one of the $y$ values results in a good signature. 
Table 6: UOV/Rainbow implementations with and without precomputation at three security categories, in CPU cycles and bytes.

\begin{tabular}{|c|c|c|c|c|c|}
\hline Scheme & Security Category & Unit & I & III & $\mathrm{V}$ \\
\hline \multirow{5}{*}{ UOV } & Sign w/o Precomp. & cycle & 201834 & 707959 & 1486775 \\
\hline & Precomp. (offline) & & 189224 & 690586 & 1460168 \\
\hline & Sign w/ Precomp. (online) & cycle & 11788 & 19439 & 23133 \\
\hline & Total (offline + online) & & 201012 & 710025 & 1483301 \\
\hline & Precomp. Memory Cost per Sig. & byte & 2256 & 5402 & 9504 \\
\hline \multirow{5}{*}{ Rainbow } & Sign w/o Precomp. & cycle & 68203 & 322799 & 807309 \\
\hline & Precomp. (offline) & & 37212 & 173204 & 508890 \\
\hline & Sign w/ Precomp. (online) & cycle & 31973 & 142179 & 278511 \\
\hline & Total (offline + online) & & 69185 & 315383 & 787401 \\
\hline & Precomp. Memory Cost per Sig. & byte & 2152 & 4792 & 5648 \\
\hline
\end{tabular}

\subsection{Resilience against Leakage or Reuse of Precomputed Values}

We have shown that precomputation dramatically improved UOV/Rainbow signing with the additional memory cost. To use the precomputed values securely, we investigate the resilience against leakage or reuse of the precomputed values in UOV and Rainbow. We show that a sufficient number of signatures generated by the precomputed values lead the full secret key recoveries of UOV and Rainbow in polynomial-time.

\subsubsection{Leakage of Precomputed Values}

Store $\left\langle s_{V}, c_{V}, L S_{V}^{-1}>\right.$ Securely. The precomputed values $<s_{V}, c_{V}, L S_{V}^{-1}>$ should be stored securely. If some precomputed values together with signatures generated by them are exposed then the secret key of UOV is completely recovered.

Wolf and Preneel [WP05] introduced the notion of equivalent keys as a fundamental tool to analyze the security of the MQ-schemes. They showed that there exist a large number of different secret keys (called equivalent keys) for a given public key in UOV and Rainbow [WP05, Tho13]. If an attacker finds any equivalent keys then it can forge any signatures on any messages without acquiring the original secret key. Key recovery attacks (KRAs) exploit the special structure of the central map, i.e. quadratic terms with zero coefficients at certain known places. Goal of the KRAs is to find an equivalent key associated to the public key. In the following theorems, we show that the leakage of precomputed values and $(n+1)$ signatures generated by the precomputed values leads to the recovery of the equivalent keys of UOV and Rainbow in polynomial-time. They can be applied to both cases of random affine secret keys and equivalent keys.

Theorem 3. If $(n+1)$ tuples $<\mathrm{m}^{(i)}, \tau^{(i)}, s_{V}^{(i)}, c_{V}^{(i)}, L S_{V}^{(i)-1}>$ are given such that the $n \times n$ matrix $\left(\sigma^{(1) \mathrm{T}} \sigma^{(2) \mathrm{T}} \cdots \sigma^{(n) \mathrm{T}}\right)$ is invertible then the secret key of UOV is completely recovered in polynomial-time.

Proof. Let $T$ be the linear part of $\mathcal{T}$ and $\mathcal{T}_{V}=\pi_{v} \circ \mathcal{T}: \mathbb{F}_{q}^{n} \mapsto \mathbb{F}_{q}^{v}$ and $T_{v}=\pi_{v} \circ T: \mathbb{F}_{q}^{n} \mapsto \mathbb{F}_{q}^{v}$, where $\pi_{v}\left(x_{1}, \cdots, x_{v}, \cdots, x_{n}\right)=\left(x_{1}, \cdots, x_{v}\right)$. By assumption, we get $T_{V}\left(\sigma^{(i)}-\sigma^{(j)}\right)=$ $\mathcal{T}_{V}\left(\sigma^{(i)}\right)-\mathcal{T}_{V}\left(\sigma^{(j)}\right)=s_{V}^{(i)}-s_{V}^{(j)}$. For each $1 \leq i \leq n$, let $\sigma_{i}=\sigma^{(i)}-\sigma^{(n+1)}$ and 
$\hat{s}_{V}^{(i)}=s_{V}^{(i)}-s_{V}^{(n+1)}$. Then we get $T_{V}\left(\sigma_{i}\right)=\hat{s}_{V}^{(i)}$ and

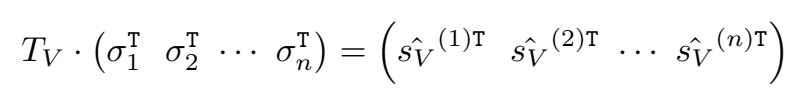

Since an $n$-dimensional diagonal matrix $\left(\begin{array}{llll}\sigma_{1}^{\mathrm{T}} & \sigma_{2}^{\mathrm{T}} & \cdots & \sigma_{n}^{\mathrm{T}}\end{array}\right)$ is invertible with high probability $\left(1-\frac{1}{q}\right)^{n}$, we get

$$
T_{V}=\left(\hat{s}_{V}^{(1) \mathrm{T}} \hat{s}_{V}^{(2) \mathrm{T}} \cdots \hat{s}_{V}^{(n) \mathrm{T}}\right) \cdot\left(\begin{array}{llll}
\sigma_{1}^{\mathrm{T}} & \sigma_{2}^{\mathrm{T}} \cdots \sigma_{n}^{\mathrm{T}}
\end{array}\right)^{-1} .
$$

It is known that there is an equivalent key $\left(\mathcal{F}^{\prime}, \mathcal{T}^{\prime}\right)$ with $\mathcal{P}=\mathcal{F}^{\prime} \circ \mathcal{T}^{\prime}$ where $\mathcal{F}^{\prime}$ has the same structure with $\mathcal{F}$ and linear coefficient matrix $T^{\prime}$ of $\mathcal{T}^{\prime}$ has the following simple form

$$
T^{\prime}=\left(\begin{array}{cc}
I^{v \times v} & T_{0}^{v \times o} \\
0^{o \times v} & I^{o \times o}
\end{array}\right)
$$

Note that the first $v$ rows of $T^{\prime}$ can be obtained by a reduced row echelon form $T_{V}$. Therefore, an equivalent key of UOV is recovered in polynomial time.

For Rainbow, we consider its equivalent keys. Let

$$
S^{-1}=\left(\begin{array}{cc}
\widetilde{S}_{1}^{o_{1} \times o_{1}} & {\widetilde{S_{2}}}^{o_{1} \times o_{2}} \\
{\widetilde{S_{3}}}^{o_{2} \times o_{1}} & {\widetilde{S_{4}}}^{o_{2} \times o_{2}}
\end{array}\right), \quad T^{-1}=\left(\begin{array}{ccc}
\widetilde{T}_{1}{ }^{v \times v} & \widetilde{T}_{2}{ }^{v \times o_{1}} & \widetilde{T}_{3}{ }^{v \times o_{2}} \\
\widetilde{T}_{4}^{o_{1} \times v} & {\widetilde{T_{5}}}^{o_{1} \times o_{1}} & {\widetilde{T_{6}}}^{o_{1} \times o_{2}} \\
\widetilde{T}_{7}{ }^{o_{2} \times v} & \widetilde{T}^{o_{2} \times o_{1}} & \widetilde{T}_{9}^{o_{2} \times o_{2}}
\end{array}\right),
$$

$S$ and $T$ are linear part of $\mathcal{S}$ and $\mathcal{T}$, respectively. For a secret key $(\mathcal{F}, \mathcal{S}, \mathcal{T})$ of Rainbow, if there exists

$$
\left(\mathcal{F}^{\prime}, \mathcal{S}^{\prime}, \mathcal{T}^{\prime}\right)=\left(\Sigma \circ \mathcal{F} \circ \Omega, \mathcal{S} \circ \Sigma^{-1}, \Omega^{-1} \circ \mathcal{T}\right)
$$

and $\Sigma \circ \mathcal{F} \circ \Omega$ and $\mathcal{F} \circ \Omega$ have the same structure for some linear maps $\Sigma \in \mathbb{G L}_{m}\left(\mathbb{F}_{q}\right)$ and $\Omega \in \mathbb{G L}_{n}\left(\mathbb{F}_{q}\right)$, then $\left(\mathcal{F}^{\prime}, \mathcal{S}^{\prime}, \mathcal{T}^{\prime}\right)$ is an equivalent key of $(\mathcal{F}, \mathcal{S}, \mathcal{T})$, where $\Sigma$ and $\Omega$ are of the form

$$
\Sigma=\left(\begin{array}{cc}
\Sigma_{1}^{o_{1} \times o_{1}} & 0^{o_{1} \times o_{2}} \\
\Sigma_{2}^{o_{2} \times o_{1}} & \Sigma_{3}^{o_{2} \times o_{2}}
\end{array}\right), \quad \Omega=\left(\begin{array}{ccc}
\Omega_{1}^{v \times v} & 0^{v \times o_{1}} & 0^{v \times o_{2}} \\
\Omega_{2}^{o_{1} \times v} & \Omega_{3}^{o_{1} \times o_{1}} & 0^{o_{1} \times o_{2}} \\
\Omega_{4}^{o_{2} \times v} & \Omega_{5}^{o_{2} \times o_{1}} & \Omega_{6}^{o_{2} \times o_{2}}
\end{array}\right)
$$

If we set submatrices of $\Sigma$ and $\Omega$ in (1) as

$$
\begin{aligned}
& \Sigma_{1}=\left(\widetilde{S_{1}}\right)^{-1}, \quad\left(\begin{array}{cc}
\Sigma_{2} & \Sigma_{3}
\end{array}\right)=\left(\begin{array}{ll}
0^{o_{2} \times o_{1}} & I^{o_{2} \times o_{2}}
\end{array}\right) \cdot S \\
& \left(\begin{array}{c}
\Omega_{1} \\
\Omega_{2} \\
\Omega_{4}
\end{array}\right)=T \cdot\left(\begin{array}{c}
I^{v \times v} \\
0^{m \times v}
\end{array}\right), \quad\left(\begin{array}{l}
\Omega_{3} \\
\Omega_{5}
\end{array}\right)=\left(\begin{array}{cc}
\widetilde{T_{5}} & \widetilde{T_{6}} \\
\widetilde{T_{8}} & \widetilde{T_{9}}
\end{array}\right)^{-1}\left(\begin{array}{l}
I^{o_{1} \times o_{1}} \\
0^{o_{2} \times o_{1}}
\end{array}\right), \quad \Omega_{6}=\left(\widetilde{T_{9}}\right)^{-1}
\end{aligned}
$$

one gets

$$
\begin{aligned}
S^{\prime-1} & =\Sigma \cdot S^{-1}=\left(\begin{array}{ccc}
I^{o_{1} \times o_{1}} & {\widetilde{S_{1}^{\prime}}}^{o_{1} \times o_{2}} \\
0^{o_{2} \times o_{1}} & I^{o_{2} \times o_{2}}
\end{array}\right) \\
T^{\prime-1} & =T^{-1} \cdot \Omega=\left(\begin{array}{ccc}
I^{v \times v} & {\widetilde{T_{1}^{\prime}}}^{v \times o_{1}} & \widetilde{T_{2}^{\prime}} v \times o_{2} \\
0^{o_{1} \times v} & I^{o_{1} \times o_{1}} & {\widetilde{T_{3}^{\prime}}}^{o_{1} \times o_{2}} \\
0^{o_{2} \times v} & 0^{o_{2} \times o_{1}} & I^{o_{2} \times o_{2}}
\end{array}\right),
\end{aligned}
$$

where $S^{\prime}$ and $T^{\prime}$ are coefficient matrices of linear part of $\mathcal{S}^{\prime}$ and $\mathcal{T}^{\prime}$, respectively [Tho13]. 
Theorem 4. If $(n+1)$ tuples $<\mathrm{m}^{(i)}, \tau^{(i)}, s_{V}^{(i)}, c_{V}^{(i)},\left(L S_{V}^{(i)}\right)^{-1}>$ are given such that the $n \times n$ matrix $\left(\sigma^{(1) \mathrm{T}} \sigma^{(2) \mathrm{T}} \cdots \sigma^{(n) \mathrm{T}}\right)$ is invertible then an equivalent key of Rainbow is completely recovered in polynomial-time.

Proof. Let $P=\left(P^{(1)}, P^{(2)}, \cdots, P^{(m)}\right)$ and $F=\left(F^{(1)}, F^{(2)}, \cdots, F^{(m)}\right)$ be the quadratic part of $\mathcal{P}$ and $\mathcal{F}$, respectively, and $S$ and $T$ are the linear part of $\mathcal{S}$ and $\mathcal{T}$, respectively. Then we have $P=S \circ F \circ T$. We denote

$$
\begin{aligned}
& O_{0}=\left\{\left(x_{1}, \cdots, x_{n}\right) \in \mathbb{F}_{q}^{n}: x_{1}=\cdots=x_{v}=0\right\}, \\
& O_{1}=\left\{\left(x_{1}, \cdots, x_{m}\right) \in \mathbb{F}_{q}^{n}: x_{1}=\cdots=x_{o_{1}}=0\right\} .
\end{aligned}
$$

We can see that if $x \in O_{0}$ then $F^{(k)}(x)=0$ for $1 \leq k \leq o_{1}$ and hence $F(x) \in O_{1}$.

Let $T_{v}=\pi_{v} \circ T: \mathbb{F}_{q}^{n} \mapsto \mathbb{F}_{q}^{v}$, where $\pi_{v}\left(x_{1}, \cdots, x_{v}, \cdots, x_{n}\right)=\left(x_{1}, \cdots, x_{v}\right)$. Similarly with the proof of Theorem 1 , we can recover $T_{V}$ if we have $n+1$ tuples in assumption. Let $\Lambda$ be the orthogonal space of the space generated by row vectors of $T_{V}$. Then if $\mathbf{b} \in \Lambda$ we get $T_{V}(\mathbf{b})=0$ and hence we get $T(\mathbf{b}) \in O_{0}$. So, we get $S^{-1} \circ P(\mathbf{b})=(F \circ T)(\mathbf{b}) \in O_{1}$. We choose $o_{2}$ vectors $\mathbf{b}_{1}, \mathbf{b}_{2}, \cdots, \mathbf{b}_{o_{2}} \in \Lambda$ and denote $\mathbf{a}_{i}=P\left(\mathbf{b}_{i}\right) \in S\left(O_{1}\right)$ for each $1 \leq i \leq o_{2}$. Then $S^{-1}\left(\mathbf{a}_{i}\right) \in O_{1}$. If $\mathbf{s}_{j}$ be $j$-th row vector of $S^{-1}$ for $1 \leq j \leq m$ then we can see that $\mathbf{a}_{i} \cdot \mathbf{s}_{j}=0$ for every $1 \leq i \leq o_{2}$ and $1 \leq j \leq o_{1}$. Let $\mathrm{S}$ be an $o_{1}$-dimensional space generated by $\mathbf{s}_{1}, \mathbf{s}_{2}, \cdots, \mathbf{s}_{o_{1}}$. For each $\mathbf{s}=\left(\mathbf{s}_{1}, \cdots, \mathbf{s}_{m}\right) \in \mathrm{S}$, we get $o_{2}$ linear equations of the form

$$
\mathbf{a}_{i} \cdot\left(\mathbf{s}_{1}, \cdots, \mathbf{s}_{m}\right)=0
$$

about $m$ variables $\mathbf{s}_{1}, \cdots, \mathbf{s}_{m}$. We solve these linear equations, and express $\mathbf{s}_{o_{1}+1}, \cdots, \mathbf{s}_{m}$ by linear combinations of $\mathbf{s}_{1}, \cdots, \mathbf{s}_{o_{1}}$ such as

$$
\begin{aligned}
\left(\begin{array}{c}
\mathbf{s}_{1} \\
\mathbf{s}_{2} \\
\vdots \\
\mathbf{s}_{O_{1}}
\end{array}\right)=\left(\begin{array}{c}
\widetilde{s}_{1, o_{1}+1} \\
\widetilde{s}_{2, o_{1}+1} \\
\vdots \\
\widetilde{s}_{O_{1}, o_{1}+1}
\end{array}\right) \mathbf{s}_{o_{1}+1}+\left(\begin{array}{c}
\widetilde{s}_{1, o_{1}+2} \\
\widetilde{s}_{2, o_{1}+2} \\
\vdots \\
\widetilde{s}_{O_{1}, o_{1}+2}
\end{array}\right) \mathbf{s}_{O_{1}+2}+\cdots+\left(\begin{array}{c}
\widetilde{s}_{1, m} \\
\widetilde{s}_{2, m} \\
\vdots \\
\widetilde{s}_{O_{1}, m}
\end{array}\right) \mathbf{s}_{m} \\
=\left(\begin{array}{cccc}
\widetilde{s}_{1, o_{1}+1} & \widetilde{s}_{1, o_{1}+2} & \cdots & \widetilde{s}_{1, m} \\
\widetilde{s}_{2, o_{1}+1} & \widetilde{s}_{2, o_{1}+2} & \cdots & \widetilde{s}_{2, m} \\
\vdots & \vdots & \ddots & \vdots \\
\widetilde{s}_{O_{1}, o_{1}+1} & \widetilde{s}_{O_{1}, o_{1}+2} & \cdots & \widetilde{s}_{O_{1}, m}
\end{array}\right)\left(\begin{array}{c}
\mathbf{s}_{o_{1}+1} \\
\mathbf{s}_{o_{1}+2} \\
\vdots \\
\mathbf{s}_{m}
\end{array}\right)=\widetilde{S}\left(\begin{array}{c}
\mathbf{s}_{o_{1}+1} \\
\mathbf{s}_{o_{1}+2} \\
\vdots \\
\mathbf{s}_{m}
\end{array}\right) .
\end{aligned}
$$

Thus, $\mathrm{S}$ is generated by row vectors of

$$
\left(\begin{array}{ll}
I^{o_{1} \times o_{1}} & \widetilde{S}
\end{array}\right) .
$$

Since the reduced row echelon form is unique, we can see that

$$
\left(\begin{array}{cc}
I^{o_{1} \times o_{1}} & \widetilde{S} \\
0^{o_{2} \times o_{1}} & I^{o_{2} \times o_{2}}
\end{array}\right)=S^{\prime-1},
$$

where $S^{\prime-1}$ is in (2). Hence, we can find $S^{\prime-1}$ in (2) in polynomial-time. The remaining part of the attack is to recover $T^{\prime-1}$ in (2). We show that we can recover $T^{\prime-1}$ by solving several linear systems. For the last $o_{2}$ columns of $T^{\prime-1}$, we can apply good key finding attack in [Tho13]. More precisely, one can find a good key $\left(\mathcal{F}^{\prime} \circ \Omega^{\prime}, \mathcal{S}^{\prime}, \mathcal{T}^{\prime \prime}\right)$ corresponding to $\left(\mathcal{F}^{\prime}, \mathcal{S}^{\prime}, \mathcal{T}^{\prime}\right)$, where $T^{\prime \prime}$, the coefficient matrix of $\mathcal{T}^{\prime \prime}$, satisfies

$$
T^{\prime \prime-1}=T^{\prime-1} \cdot \Omega^{\prime}=\left(\begin{array}{cccc}
I^{v \times v} & 0^{v \times o_{1}} & 0^{v \times\left(o_{2}-1\right)} & {\widetilde{T_{2}^{\prime \prime}}}^{v \times 1} \\
0^{o_{1} \times v} & I^{o_{1} \times o_{1}} & 0^{o_{1} \times\left(o_{2}-1\right)} & {\widetilde{T_{3}^{\prime \prime}}}_{o_{1} \times 1} \\
0^{\left(o_{2}-1\right) \times v} & 0^{\left(o_{2}-1\right) \times o_{1}} & I^{\left(o_{2}-1\right) \times\left(o_{2}-1\right)} & 0^{\left(o_{2}-1\right) \times 1} \\
0^{1 \times v} & 0^{1 \times o_{1}} & 0^{1 \times\left(o_{2}-1\right)} & 1
\end{array}\right),
$$


if we set

$$
\Omega^{\prime}=\left(\begin{array}{cc}
T_{n, n-1}^{\prime} & 0^{(n-1) \times 1} \\
1
\end{array}\right),
$$

where $T_{n, n-1}^{\prime}$ is the $n \times(n-1)$ submatrix of $T^{\prime}$ removing the last column from $T^{\prime}$. Note that $\left(\frac{\widetilde{T_{2}^{\prime \prime}}}{\widetilde{T_{3}^{\prime \prime}}}\right)$ is identical to the $\left(v+o_{1}\right) \times 1$ submatrix of $\left(\begin{array}{c}\widetilde{T_{2}^{\prime}} \\ \widetilde{T_{3}^{\prime}}\end{array}\right)$ consisting of the last column. Then we get a multivariate system to find this equivalent key that consists of the following form of equations

$$
F_{i, j}^{\prime \prime(k)}=\sum_{a=1}^{m} \sum_{b=1}^{n} \sum_{c=b}^{n} P_{b, c}^{(a)} \widetilde{s_{k, a}^{\prime \prime}} \widetilde{t_{b, i}^{\prime \prime}} \widetilde{t_{c, j}^{\prime \prime}}
$$

where $F^{\prime \prime(k)}=\left(F_{i, j}^{\prime \prime(k)}\right)_{i, j}$ is the symmetric coefficient matrix of quadratic part of $\mathcal{F}^{\prime \prime(k)}$ for $1 \leq k \leq m$ and $S^{\prime \prime-1}=\left(\widetilde{s_{i, j}^{\prime \prime}}\right)_{i, j}, T^{\prime \prime-1}=\left(\widetilde{t_{i, j}^{\prime \prime}}\right)_{i, j}$. We get $F_{i, n}^{\prime \prime(k)}=0$ for $1 \leq i \leq n-1$ and $1 \leq k \leq o_{1}$. More precisely, we get $o_{1}(n-1)$ equations of the following form

$$
F_{i, n}^{\prime \prime(k)}=\sum_{a=o_{1}+1}^{m} \sum_{b=1}^{v+o_{1}} P_{b, n}^{(a)} \widetilde{s_{k, a}^{\prime}} \widetilde{t_{b, n}^{\prime}}+\sum_{a=o_{1}+1}^{m} P_{n, n}^{(a)} \widetilde{s_{k, a}^{\prime}}+\sum_{b=1}^{v+o_{1}} P_{b, n}^{(k)} \widetilde{t_{b, n}^{\prime}}+P_{n, n}^{(k)} .
$$

Since we have already recovered all entries of $S^{\prime-1}$, all obtained equations are linear about $\widetilde{t_{b, n}^{\prime}}$. So, we have a linear system of $o_{1}(n-1)$ equations with $v+o_{1}$ variables which can be solvable in polynomial time. Now, we need to recover $\widetilde{T_{1}^{\prime}}$ in $(2)$. We consider equations of the form

$$
F_{i, j}^{\prime(k)}=\sum_{a=1}^{m} \sum_{b=1}^{n} \sum_{c=b}^{n} P_{b, c}^{(a)} \widetilde{s_{k, a}^{\prime}} \widetilde{t_{b, i}^{\prime}} \widetilde{t_{c, j}^{\prime}}
$$

which can be obtained from $F=S^{\prime-1} \circ P \circ T^{-1}$, where $v+o_{1}+1 \leq j \leq n$ and $1 \leq k \leq o_{1}$. Since we already recover all entries of $S^{\prime-1}$ and $\widetilde{t_{c, j}^{\prime}}$, we get $o_{1} o_{2}$ linear equations with $v$ variables. For suggested parameters, we get enough equations to recover these variables. Therefore, we can totally recover an equivalent key by solving several linear systems.

\subsubsection{Reuse of Precomputed Values}

Do not Reuse $<s_{V}, c_{V}, L S_{V}^{-1}>$. The precomputed value $<s_{V}, c_{V}, L S_{V}^{-1}>$ should not be reused in signing.

Recently, Shim and Koo [SK20] showed that the equivalent key of UOV is completely recovered in polynomial-time from $(m+1)$ signatures generated by the reused Vinegar values. However, they did not show a polynomial-time recovery of Rainbow's equivalent key. Their results are given the following Theorem 5.

Theorem 5. [SK20] If $(m+1)$ signatures generated by the reused Vinegar values are given then

- the equivalent key of UOV is completely recovered in polynomial time,

- the complexity of the KRAs using good keys on Rainbow is determined by solving a multivariate system of $m$ quadratic equations with $o_{1}$ variables.

Now, we provide a more improved analysis: $\left(o_{2}+1\right)$ signatures generated by the fixed Vinegar values lead to the full secret key recovery of Rainbow in polynomial-time. 
Theorem 6. If $\left(o_{2}+1\right)$ signatures generated by reusing the precomputed values then an equivalent key of Rainbow is recovered in polynomial-time with high probability.

Proof. As in Theorem 2, we define $P=\left(P^{(1)}, P^{(2)}, \cdots, P^{(m)}\right), F=\left(F^{(1)}, F^{(2)}, \cdots, F^{(m)}\right)$, $S, T, O_{0}$, and $O_{1}$. We can see that if $x \in O_{0}$ then $F^{(k)}(x)=0$ for $1 \leq k \leq o_{1}$ and thus $F(x) \in O_{1}$. Suppose that $\left(o_{2}+1\right)$ signatures $\left(\sigma^{(1)}, \cdots, \sigma^{\left(o_{2}+1\right)}\right)$ are generated by the precomputed values. The probability of getting $\left(o_{2}+1\right)$ signatures generated by the fixed Vinegar vector $s_{V}$ is about

$$
\left(1-\frac{1}{q}\right)^{o_{2}} \text {. }
$$

Define $T_{v}=\pi_{v} \circ T: \mathbb{F}_{q}^{n} \mapsto \mathbb{F}_{q}^{v}$, where $\pi_{v}\left(x_{1}, \cdots, x_{v}, \cdots, x_{n}\right)=\left(x_{1}, \cdots, x_{v}\right)$. Then, we get $T_{v}\left(\sigma^{(i)}\right)=T_{v}\left(\sigma^{(j)}\right)$ for $1 \leq i<j \leq o_{2}+1$ with high probability. Since $T_{v}$ is a linear transformation, we get

$$
T_{v}\left(\sigma^{(i)}-\sigma^{(j)}\right)=T_{v}\left(\sigma^{(i)}\right)-T_{v}\left(\sigma^{(j)}\right)=0,
$$

for $1 \leq i<j \leq o_{2}+1$. Define $\mathbf{b}_{i}=\sigma^{(1)}-\sigma^{(i+1)}$ for $1 \leq i \leq o_{2}$. Then $T_{v}\left(\mathbf{b}_{i}\right)=0$ for $1 \leq i \leq m$, and $T\left(\mathbf{b}_{i}\right) \in O_{0}$. Thus, $(F \circ T)\left(\mathbf{b}_{i}\right) \in O_{1}$ and $S^{-1} \circ P\left(\mathbf{b}_{i}\right) \in O_{1}$ for all $1 \leq i \leq o_{2}$. Let $\mathbf{a}_{i}=P\left(\mathbf{b}_{i}\right) \in S\left(O_{1}\right)$ for each $1 \leq i \leq o_{2}$, then $S^{-1}\left(\mathbf{a}_{i}\right) \in O_{1}$. If $\mathbf{s}_{j}$ be the $j$-th row vector of $S^{-1}$ for $1 \leq j \leq m$ then we can see that $\mathbf{a}_{i} \cdot \mathbf{s}_{j}=0$ for every $1 \leq i \leq o_{2}$ and $1 \leq j \leq o_{1}$. Let $\mathbf{S}$ be an $o_{1}$-dimensional space generated by $\mathbf{s}_{1}, \mathbf{s}_{2}, \cdots, \mathbf{s}_{o_{1}}$. For each $\mathbf{s}=\left(\mathbf{s}_{1}, \cdots, \mathbf{s}_{m}\right) \in \mathrm{S}$, we get $o_{2}$ linear equations of the form

$$
\mathbf{a}_{i} \cdot\left(\mathbf{s}_{1}, \cdots, \mathbf{s}_{m}\right)=0
$$

about $m$ variables $\mathbf{s}_{1}, \cdots, \mathbf{s}_{m}$. The rest of this proof is the same as that of Theorem 2 . Therefore, an equivalent key of Rainbow is completely recovered in polynomial-time.

Note that the results presented in the above theorems are determined by leakage or reused Vinegar values together with signatures generated by the Vinegar values. Other values in the precomputed values except the Vinegar values do not affect the attacks in the theorems.

Zambonin et al.'s Variants of Rainbow. At Africacrypt 2019, Zambonin et al. [ZBC19] proposed three variants of Rainbow to shorten the secret key size by using fixed Vinegar values and central polynomials substituted by the fixed Vinegar values in key generations. In other words, the secret key includes the fixed Vinegar values and all the signatures are generated by the same Vinegar values in their scheme. Theorem 6 shows that their three variants are entirely broken by the key recovery attacks using good keys.

Applicability of Our Optimization to Cyclic/Compressed Versions of UOV and Rainbow. The signing processes of Cyclic versions of UOV and Rainbow are identical to those of the original versions. The signing processes of their Compressed versions are identical to those of the original versions except that the Compressed versions require additional secret key recoveries before generating a signature. Thus, our BMI method and precomputation can be applied to signing in Cyclic/Compressed versions of UOV and Rainbow.

Comparison. We summarize the overall overview of UOV/Rainbow including our optimizations in Table 7. In the Table, Comp., Precomp., Mem., M-V prod., subst., Inv., and $\mathrm{PV}$ are abbreviations of computation precomputation, memory, matrix-vector product, substitution, inversion, and precomputed values, respectively. Online Comp. and Mem. Cost represents the computation required to generate a signature with the precomputed values in online phase and the memory cost required for precomputation per signature, respectively. 
Table 7: Comparison of UOV/Rainbow with our optimizations.

\begin{tabular}{|c|c|c|c|c|}
\hline \multicolumn{3}{|c|}{ Scheme } & UOV & Rainbow \\
\hline \multirow{4}{*}{\multicolumn{3}{|c|}{$\begin{array}{l}\text { Structure } \\
\text { Public Key } \\
\text { Hard Problems } \\
\text { Invert }\end{array}$}} & ASA, Single Layer & ASA, Two Layers \\
\hline & & & $\mathcal{P}=\mathcal{F} \circ \mathcal{T}$ & $\mathcal{P}=\mathcal{S} \circ \mathcal{F} \circ \mathcal{T}$ \\
\hline & & & MQ, EIP & MQ, EIP, MinRank \\
\hline & & & Oil-Vinegar Method & Oil-Vinegar Method \\
\hline \multirow{6}{*}{$\begin{array}{l}\text { Solving } \\
\text { Linear } \\
\text { Systems }\end{array}$} & \multirow{3}{*}{$\begin{array}{l}\text { Sign } \\
(\mathrm{GE})\end{array}$} & $\mathrm{I}$ & 201834 cycles & 68203 cycles \\
\hline & & III & 707959 cycles & 322799 cycles \\
\hline & & $\mathrm{V}$ & 1486775 cycles & 807309 cycles \\
\hline & \multirow{3}{*}{$\begin{array}{l}\text { Sign } \\
(\mathrm{BMI})\end{array}$} & I & 176884 cycles & - \\
\hline & & III & 563519 cycles & 270731 cycles \\
\hline & & $\mathrm{V}$ & 981351 cycles & 650400 cycles \\
\hline \multirow{6}{*}{ Precomp. } & \multicolumn{2}{|l|}{ PV } & $\left(s_{V}, c_{V}, L S_{V}^{-1}\right)$ & $\begin{array}{l}\left(s_{V, 1}, c_{V, 1}, L S_{V, 1}^{-1},\right. \\
\left.\left\{\mathcal{F}^{\left(o_{1}+i\right)}\left(s_{V}\right)\right\}_{i=1}^{o_{2}}\right)\end{array}$ \\
\hline & \multicolumn{2}{|l|}{$\begin{array}{l}\text { Online } \\
\text { Comp. }\end{array}$} & Two M-V prod. & $\begin{array}{l}\text { Subst. of } o_{1} \text {-values, } \\
\text { One Inv., } \\
\text { Three M-V prod. }\end{array}$ \\
\hline & \multicolumn{2}{|l|}{$\begin{array}{l}\text { Mem. } \\
\text { Cost }\end{array}$} & $2 v+m^{2}$ bytes & $2 v+o_{1}^{2}+\left(o_{2}+1\right) o_{2}$ bytes \\
\hline & \multirow{3}{*}{$\begin{array}{l}\text { Sign w/ } \\
\text { Precomp. }\end{array}$} & $\mathrm{I}$ & 11968 cycles & 32129 cycles \\
\hline & & III & 19968 cycles & 144735 cycles \\
\hline & & $\mathrm{V}$ & 23667 cycles & 288008 cycles \\
\hline \multirow{2}{*}{ Resistant } & \multirow{2}{*}{\multicolumn{2}{|c|}{$\begin{array}{l}\text { PV Leakage } \\
\text { PV Reuse }\end{array}$}} & Insecure $(n+1)$ & Insecure $(n+1)$ \\
\hline & & & Insecure $(m+1)$ & Insecure $\left(o_{2}+1\right)$ \\
\hline
\end{tabular}

\section{Conclusion}

We present efficient implementations of UOV and Rainbow by using new optimizations for signing to accelerate solving linear systems and the Vinegar value substitution. In our optimizations, the larger the size of a matrix being inverted, the greater the performance improvement and the higher the security level, the greater the effect of the optimizations. Precomputations of UOV and Rainbow improve their signing performance. The secure use of the precomputation methods requires careful implementations that do not reuse the Vinegar values and do not expose the precomputed values. The potential risks can be prevented by deleting the Vinegar values and the precomputed values immediately after using them.

\section{References}

$\left[\mathrm{ABC}^{+} 20\right]$ Martin R. Albrecht, Daniel J. Bernstein, Tung Chou, Carlos Cid, Jan Gilcher, Tanja Lange, Varun Maram, Ingo von Maurich, Rafael Misoczki, Ruben Niederhagen, Kenneth G. Paterson, Edoardo Persichetti, Christiane Peters, Peter Schwabe, Nicolas Sendrier, Jakub Szefer, Cen Jung Tjhai, Martin Tomlinson, and Wen Wang. Classic McEliece. Technical report, National Institute of Standards and Technology, 2020. available at https : //csrc . nist. gov/projects/post-quantum-cryptography/round-3-submissions. 
$\left[\mathrm{BBB}^{+} 20\right]$ Magali Bardet, Pierre Briaud, Maxime Bros, Philippe Gaborit, Vincent Neiger, Olivier Ruatta, and Jean-Pierre Tillich. An algebraic attack on rank metric code-based cryptosystems. In Anne Canteaut and Yuval Ishai, editors, EUROCRYPT 2020, Part III, volume 12107 of LNCS, pages 64-93. Springer, Heidelberg, May 2020.

$\left[\mathrm{BBC}^{+} 20\right]$ Magali Bardet, Maxime Bros, Daniel Cabarcas, Philippe Gaborit, Ray A. Perlner, Daniel Smith-Tone, Jean-Pierre Tillich, and Javier A. Verbel. Improvements of algebraic attacks for solving the rank decoding and MinRank problems. In Shiho Moriai and Huaxiong Wang, editors, ASIACRYPT 2020, Part I, volume 12491 of $L N C S$, pages 507-536. Springer, Heidelberg, December 2020.

[BERW08] Andrey Bogdanov, Thomas Eisenbarth, Andy Rupp, and Christopher Wolf. Time-area optimized public-key engines: Cryptosystems as replacement for elliptic curves? In Elisabeth Oswald and Pankaj Rohatgi, editors, CHES 2008, volume 5154 of LNCS, pages 45-61. Springer, Heidelberg, August 2008.

[Beu20] Ward Beullens. Improved cryptanalysis of UOV and rainbow. Cryptology ePrint Archive, Report 2020/1343, 2020. https://eprint.iacr.org/2020/ 1343.

$\left[\mathrm{CCC}^{+}\right.$09] Anna Inn-Tung Chen, Ming-Shing Chen, Tien-Ren Chen, Chen-Mou Cheng, Jintai Ding, Eric Li-Hsiang Kuo, Frost Yu-Shuang Lee, and Bo-Yin Yang. SSE implementation of multivariate PKCs on modern x86 CPUs. In Christophe Clavier and Kris Gaj, editors, CHES 2009, volume 5747 of LNCS, pages 33-48. Springer, Heidelberg, September 2009.

$\left[\mathrm{CDH}^{+} 20\right]$ Cong Chen, Oussama Danba, Jeffrey Hoffstein, Andreas Hulsing, Joost Rijneveld, John M. Schanck, Peter Schwabe, William Whyte, Zhenfei Zhang, Tsunekazu Saito, Takashi Yamakawa, and Keita Xagawa. NTRU. Technical report, National Institute of Standards and Technology, 2020. available at https://csrc.nist.gov/projects/post-quantum-cryptography/ round-3-submissions.

[CHT12] Peter Czypek, Stefan Heyse, and Enrico Thomae. Efficient implementations of MQPKS on constrained devices. In Emmanuel Prouff and Patrick Schaumont, editors, CHES 2012, volume 7428 of LNCS, pages 374-389. Springer, Heidelberg, September 2012.

$\left[\mathrm{DCP}^{+} 19\right]$ Jintai Ding, Ming-Shing Chen, Albrecht Petzoldt, Dieter Schmidt, and BoYin Yang. Rainbow. Technical report, National Institute of Standards and Technology, 2019. available at https://csrc.nist.gov/projects/ post-quantum-cryptography/round-2-submissions.

$\left[\mathrm{DCP}^{+} 20 \mathrm{a}\right]$ Jintai Ding, Ming-Shing Chen, Albrecht Petzoldt, Dieter Schmidt, Bo-Yin Yang, Matthias Kannwischer, and Jacques Patarin. Modified parameters of Rainbow in response to a refined analysis of the Rainbow band separation attack by the NIST team and the recent new MinRank attacks. Technical report, Rainbow Team, 2020. available at https://troll.iis.sinica.edu. tw/by-publ/recent/rainbow-pars.pdf.

$\left[\mathrm{DCP}^{+} 20 \mathrm{~b}\right]$ Jintai Ding, Ming-Shing Chen, Albrecht Petzoldt, Dieter Schmidt, Bo-Yin Yang, Matthias Kannwischer, and Jacques Patarin. Rainbow. Technical report, National Institute of Standards and Technology, 2020. available at https://csrc.nist.gov/projects/post-quantum-cryptography/ round-3-submissions. 
$\left[\mathrm{DCP}^{+} 20 \mathrm{c}\right]$ Jintai Ding, Ming-Shing Chen, Albrecht Petzoldt, Dieter Schmidt, Bo-Yin Yang, Matthias Kannwischer, and Jacques Patarin. Response to recent paper by Ward Beullens. Technical report, Rainbow Team, 2020. available at https: //troll.iis.sinica.edu.tw/by-publ/recent/response-ward.pdf.

$\left[\mathrm{DKR}^{+} 20\right]$ Jan-Pieter D'Anvers, Angshuman Karmakar, Sujoy Sinha Roy, Frederik Vercauteren, Jose Maria Bermudo Mera, Michiel Van Beirendonck, and Andrea Basso. SABER. Technical report, National Institute of Standards and Technology, 2020. available at https://csrc.nist.gov/projects/ post-quantum-cryptography/round-3-submissions.

[DS05] Jintai Ding and Dieter Schmidt. Rainbow, a new multivariable polynomial signature scheme. In John Ioannidis, Angelos Keromytis, and Moti Yung, editors, ACNS 05, volume 3531 of $L N C S$, pages 164-175. Springer, Heidelberg, June 2005.

[EGM90] Shimon Even, Oded Goldreich, and Silvio Micali. On-line/off-line digital schemes. In Gilles Brassard, editor, CRYPTO'89, volume 435 of LNCS, pages 263-275. Springer, Heidelberg, August 1990.

[KPG99] Aviad Kipnis, Jacques Patarin, and Louis Goubin. Unbalanced oil and vinegar signature schemes. In Jacques Stern, editor, EUROCRYPT'99, volume 1592 of LNCS, pages 206-222. Springer, Heidelberg, May 1999.

$\left[\mathrm{LDK}^{+} 20\right]$ Vadim Lyubashevsky, Léo Ducas, Eike Kiltz, Tancrède Lepoint, Peter Schwabe, Gregor Seiler, Damien Stehlé, and Shi Bai. CRYSTALS-DILITHIUM. Technical report, National Institute of Standards and Technology, 2020. available at https://csrc.nist.gov/projects/post-quantum-cryptography/ round-3-submissions.

[MI88] Tsutomu Matsumoto and Hideki Imai. Public quadratic polynominal-tuples for efficient signature-verification and message-encryption. In C. G. Günther, editor, EUROCRYPT'88, volume 330 of $L N C S$, pages 419-453. Springer, Heidelberg, May 1988.

$\left[\mathrm{ND}^{+} 77\right]$ Ben Noble, James W Daniel, et al. Applied linear algebra, volume 3. PrenticeHall Englewood Cliffs, NJ, 1977.

[Pat96] Jacques Patarin. Hidden fields equations (HFE) and isomorphisms of polynomials (IP): Two new families of asymmetric algorithms. In Ueli M. Maurer, editor, EUROCRYPT'96, volume 1070 of LNCS, pages 33-48. Springer, Heidelberg, May 1996.

[PCG01] Jacques Patarin, Nicolas Courtois, and Louis Goubin. QUARTZ, 128-bit long digital signatures. In David Naccache, editor, CT-RSA 2001, volume 2020 of LNCS, pages 282-297. Springer, Heidelberg, April 2001.

$\left[\mathrm{PCY}^{+} 15\right] \quad$ Albrecht Petzoldt, Ming-Shing Chen, Bo-Yin Yang, Chengdong Tao, and Jintai Ding. Design principles for HFEv-based multivariate signature schemes. In Tetsu Iwata and Jung Hee Cheon, editors, ASIACRYPT 2015, Part I, volume 9452 of $L N C S$, pages 311-334. Springer, Heidelberg, November / December 2015.

$\left[\mathrm{PFH}^{+} 20\right]$ Thomas Prest, Pierre-Alain Fouque, Jeffrey Hoffstein, Paul Kirchner, Vadim Lyubashevsky, Thomas Pornin, Thomas Ricosset, Gregor Seiler, William Whyte, and Zhenfei Zhang. FALCON. Technical report, National Institute of Standards and Technology, 2020. available at https://csrc.nist.gov/ projects/post-quantum-cryptography/round-3-submissions. 
[PS20] Ray Perlner and Daniel Smith-Tone. Rainbow band separation is better than we thought. Cryptology ePrint Archive, Report 2020/702, 2020. https: //eprint.iacr.org/2020/702.

[RGCB19] Prasanna Ravi, Sourav Sen Gupta, Anupam Chattopadhyay, and Shivam Bhasin. Improving speed of Dilithium's signing procedure. Cryptology ePrint Archive, Report 2019/420, 2019. https://eprint.iacr.org/2019/420.

$\left[\mathrm{SAB}^{+} 20\right]$ Peter Schwabe, Roberto Avanzi, Joppe Bos, Léo Ducas, Eike Kiltz, Tancrède Lepoint, Vadim Lyubashevsky, John M. Schanck, Gregor Seiler, and Damien Stehlé. CRYSTALS-KYBER. Technical report, National Institute of Standards and Technology, 2020. available at https://csrc.nist.gov/ projects/post-quantum-cryptography/round-3-submissions.

[SK20] Kyung-Ah Shim and Namhun Koo. Algebraic fault analysis of UOV and Rainbow with the leakage of random vinegar values. IEEE Trans. Inf. Forensics Secur., 15:2429-2439, 2020.

[SSH11] Koichi Sakumoto, Taizo Shirai, and Harunaga Hiwatari. On provable security of UOV and HFE signature schemes against chosen-message attack. In BoYin Yang, editor, Post-Quantum Cryptography - 4th International Workshop, PQCrypto 2011, pages 68-82. Springer, Heidelberg, November / December 2011.

[Str69] Volker Strassen. Gaussian elimination is not optimal. Numerische mathematik, 13(4):354-356, 1969.

[Tho13] Enrico Thomae. About the security of multivariate quadratic public key schemes. PhD thesis, Ruhr-Universit Ãdt Bochum, 2013.

[VBC $\left.{ }^{+} 19\right]$ Javier A. Verbel, John Baena, Daniel Cabarcas, Ray A. Perlner, and Daniel Smith-Tone. On the complexity of "superdetermined" minrank instances. In Jintai Ding and Rainer Steinwandt, editors, Post-Quantum Cryptography - 10th International Conference, PQCrypto 2019, pages 167-186. Springer, Heidelberg, 2019.

[WP05] Christopher Wolf and Bart Preneel. Large superfluous keys in multivariate quadratic asymmetric systems. In Serge Vaudenay, editor, PKC 2005, volume 3386 of $L N C S$, pages 275-287. Springer, Heidelberg, January 2005.

[WWCJ10] Jason M. Weaver, Kristin L. Wood, Richard H. Crawford, and Dan Jensen. Design of energy harvesting technology: Feasibility for low-power wireless sensor networks. In International Design Engineering Technical Conferences and Computers and Information in Engineering Conference, volume 44144, pages $463-472,2010$.

[ZBC19] Gustavo Zambonin, Matheus S. P. Bittencourt, and Ricardo Felipe Custódio. Handling vinegar variables to shorten Rainbow key pairs. In Johannes Buchmann, Abderrahmane Nitaj, and Tajje eddine Rachidi, editors, AFRICACRYPT 19, volume 11627 of $L N C S$, pages 391-408. Springer, Heidelberg, July 2019. 